\title{
Universality of an Impurity in a Bose-Einstein Condensate
}

\author{
Shuhei M. Yoshida, ${ }^{1,2}$ Shimpei Endo, ${ }^{2}$ Jesper Levinsen, ${ }^{2}$ and Meera M. Parish ${ }^{2}$ \\ ${ }^{1}$ Department of Physics, The University of Tokyo, Tokyo 113-0033, Japan \\ ${ }^{2}$ School of Physics and Astronomy, Monash University, Victoria 3800, Australia
}

(Received 13 October 2017; revised manuscript received 12 December 2017; published 13 February 2018)

\begin{abstract}
We consider the ground-state properties of an impurity particle ("polaron") resonantly interacting with a Bose-Einstein condensate (BEC). Focusing on the equal-mass system, we use a variational wave function for the polaron that goes beyond previous work and includes up to three Bogoliubov excitations of the BEC, thus allowing us to capture both Efimov trimers and associated tetramers. We find that the length scale associated with Efimov trimers (i.e., the three-body parameter) can strongly affect the polaron's behavior, even at densities where there are no well-defined Efimov states. However, by comparing our results with recent quantum Monte Carlo calculations, we argue that the polaron energy is a universal function of the Efimov three-body parameter for sufficiently low boson densities. We further support this conclusion by showing that the energies of the deepest bound Efimov trimers and tetramers at unitarity are universally related to one another, regardless of the microscopic model. On the other hand, we find that the quasiparticle residue and effective mass sensitively depend on the coherence length $\xi$ of the BEC, with the residue tending to zero as $\xi$ diverges, in a manner akin to the orthogonality catastrophe.
\end{abstract}

DOI: 10.1103/PhysRevX.8.011024

\section{INTRODUCTION}

Universality is a powerful concept in physics, allowing one to construct physical descriptions of systems that are independent of the precise microscopic details or energy scales. A prime example is the dilute two-component Fermi gas at unitarity [1], where the scattering length $a \rightarrow \pm \infty$ and the interparticle spacing greatly exceed the range of the interactions. Here, the large separation of length scales means that the unitary Fermi gas is independent of an interaction length scale, thus resulting in universal properties that describe systems ranging from atomic gases at microkelvin temperatures to the inner crust of neutron stars. Indeed, highly controlled cold-atom experiments have successfully determined its universal equation of state [2-4], pairing correlations [5-12], and transport properties [13]. More recently, cold-atom experiments have started to probe Bose gases in the unitary regime [14-20]. Even though the strongly interacting Bose gas is expected to be unstable, there are regimes where the system appears to be sufficiently long-lived to observe well-defined static and dynamical properties $[14,16]$. However, it remains an open

Published by the American Physical Society under the terms of the Creative Commons Attribution 4.0 International license. Further distribution of this work must maintain attribution to the author(s) and the published article's title, journal citation, and DOI.

\author{
Subject Areas: Atomic and Molecular Physics, \\ Condensed Matter Physics, \\ Quantum Physics
}

question whether the unitary Bose system can also display universal behavior that has relevance to a range of different physical systems.

In contrast to the Fermi case, bosonic systems at unitarity always support few-body Efimov bound states [21-26], whose energy and size depend on short-distance parameters such as the interaction range $r$. Thus, there is an additional interaction length scale that can impact the behavior of the unitary Bose system and potentially make it sensitive to microscopic details. Indeed, the deepest bound trimers and larger few-body clusters typically cannot be universally related to each other without invoking a specific model for the short-range interactions $[24,25]$.

To investigate the universality of a Bose system at unitarity, we consider an impurity immersed in a BoseEinstein condensate (BEC), where the boson-impurity interactions can be tuned to unitarity while the rest of the system remains weakly interacting. This so-called "Bose polaron" has received much theoretical attention [27-41] and has very recently been observed in cold-atom experiments $[15,16]$. Moreover, it has been extended to impurities with more complex internal structure [42-44], and it has potential relevance to polaron problems in solidstate systems-for instance, the limit of weak impurityboson interactions can be directly mapped to the Fröhlich model $[27,45]$. For stronger interactions, two recent works investigated the possibility of coupling the polaron to Efimov trimers $[32,40]$. However, there has yet to be a 


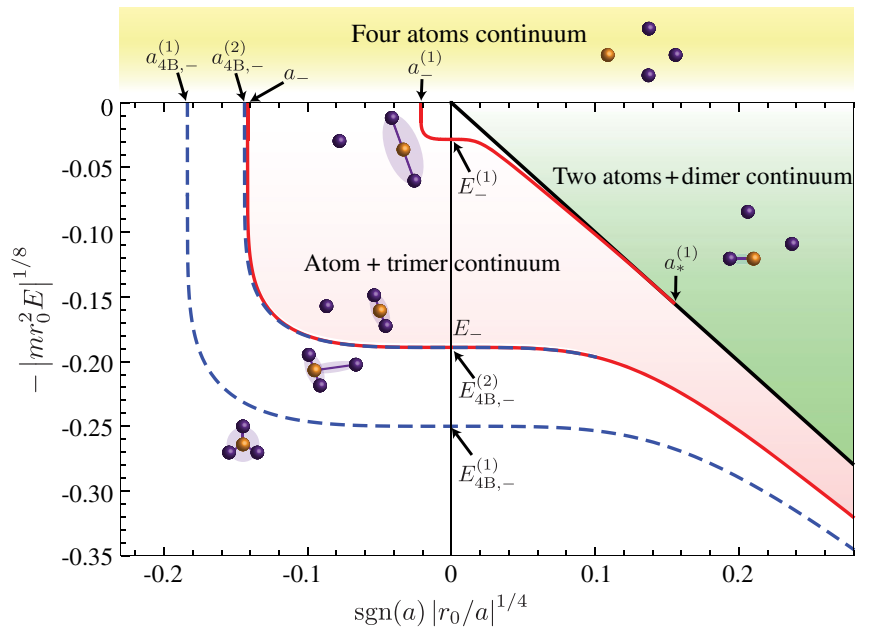

FIG. 1. Few-body energy spectrum calculated from Eqs. (7)(9). The ground (first excited) trimer is shown as a red solid curve, which first appears at a negative scattering length $a_{-}\left(a_{-}^{(1)}\right)$ and then dissociates into the atom-dimer continuum (delimited by the black solid line) at a positive scattering length $a_{*}\left(a_{*}^{(1)}\right)$, where $a_{*}$ is outside the plotted region. We only show the ground and first excited trimers, but there exists an infinite series of higher excited trimers with an accumulation point at unitarity, $1 / a=0$. We also find two tetramer states (blue dashed lines) tied to the ground Efimov trimer. The excited tetramer is very weakly bound, but it persists at unitarity and disappears into the atom-trimer continuum at the positive scattering length $a \simeq 9 \times 10^{3}\left|r_{0}\right|$.

systematic study of the effect of Efimov physics on the Bose polaron at unitarity.

The Bose polaron is a promising system to search for universal behavior since the Efimov trimers consisting of the impurity and two bosons are orders of magnitude larger than the short-distance scale $r$ (provided the impurity mass is not too small) $[46,47]$. If we parametrize the size of the deepest Efimov trimer by $\left|a_{-}\right|$, where $a_{-}$is the scattering length at which the trimer crosses the three-atom continuum (see Fig. 1), then the hierarchy of length scales at unitarity is $r \ll n^{-1 / 3} \ll\left|a_{-}\right|$for the typical densities $n$ in experiment $[15,16]$. This suggests that there is a regime where Efimov physics is irrelevant, such that the groundstate properties of the polaron only depend on the density, like in the case of an impurity resonantly interacting with a Fermi gas [48-52].

In this paper, we address this question using impurities that have a mass equal to that of the bosons-a situation that has already been realized in the ${ }^{39} \mathrm{~K}$ atomic gas experiments in Ref. [16]. We first investigate the few-body limit and determine the Efimov trimer and associated tetramer states that involve the impurity. For vanishing boson-boson interactions, we show that the ratios of the binding energies for the deepest bound states are universal, unlike the case of three and four resonantly interacting identical bosons. We then include this Efimov physics in the many-body system by constructing a variational wave function for the ground-state Bose polaron which recovers both three- and four-body equations in the limit of zero density. Strikingly, we find that the polaron properties at unitarity sensitively depend on the Efimov scale $\left|a_{-}\right|$, even in the regime where $\left|a_{-}\right|$far exceeds the interparticle spacing. However, we show that the ground-state polaron energy is a universal function of $n^{1 / 3}\left|a_{-}\right|$that is model independent in the regime $n^{1 / 3} r \ll 1$. We corroborate this finding by comparing our results with recent quantum Monte Carlo (QMC) calculations [33,36].

In the nonuniversal, high-density limit $n^{1 / 3} r \gg 1$, we consider the case of a narrow Feshbach resonance, and we derive perturbative expressions for the polaron energy and contact at unitarity. This allows us to demonstrate, for the first time, that the Bose polaron at unitarity can be thermodynamically stable even in the limit of vanishing boson-boson interactions. On the other hand, we find that the quasiparticle residue and effective mass sensitively depend on the BEC coherence length. For the case of an ideal BEC, the effective mass converges to a finite value as we increase the number of excitations of the condensate, while the residue vanishes in a manner reminiscent of the orthogonality catastrophe for a static impurity in a Fermi gas [53].

\section{MODEL AND VARIATIONAL APPROACH}

We consider an impurity atom immersed in a weakly interacting homogeneous Bose-Einstein condensate at zero temperature. The interactions in the medium are characterized by the boson-boson scattering length $a_{B}$, and we thus require $n_{0} a_{B}^{3} \ll 1$, with $n_{0}$ the condensate density (which essentially equals the total density $n$ in this regime). This allows us to treat both the ground state and the excitations of the BEC within Bogoliubov theory [54]. Note that we always implicitly assume $a_{B}>0$ to ensure the stability of the condensate.

The presence of the impurity in the medium adds two length scales associated with the impurity-boson interaction. The first of these is the scattering length $a$ between the impurity and a boson from the medium. We disregard this length scale since we focus on the strong-coupling unitary regime close to a Feshbach resonance, where $|a|$ greatly exceeds all other length scales; i.e., we generally consider $1 / a=0$. Here, we assume short-range contact interactions, a scenario that is well realized in current cold-atom experiments.

The second additional length scale is the so-called threebody parameter, which characterizes the size of the smallest (ground-state) Efimov trimer consisting of two bosons and the impurity. To demonstrate the model independence of our results, we fix this Efimov length scale in two different ways:

(i) $r_{0}$ model-We introduce an effective range $r_{0}$, which directly relates the three-body parameter to the two-body interaction [55]. 
(ii) $\Lambda$ model-We take $r_{0} \rightarrow 0$ and instead apply an explicit ultraviolet cutoff $\Lambda$ to the momenta involved in Efimov physics, which is equivalent to including a three-body repulsion [56].

As we explicitly show in Sec. III, for an impurity of the same mass as the medium atoms - the scenario considered here-the precise manner of regularizing Efimov physics is unimportant, owing to the large separation of scales between the short-range physics and Efimov physics.

Given the above considerations, we model the system using a two-channel description of the Feshbach resonance [57], which corresponds to the Hamiltonian (setting $\hbar$ and the volume to 1 ):

$$
\begin{aligned}
\hat{H}= & \sum_{\mathbf{k}}\left[E_{\mathbf{k}} \beta_{\mathbf{k}}^{\dagger} \beta_{\mathbf{k}}+\epsilon_{\mathbf{k}} c_{\mathbf{k}}^{\dagger} c_{\mathbf{k}}+\left(\epsilon_{\mathbf{k}}^{d}+\nu_{0}\right) d_{\mathbf{k}}^{\dagger} d_{\mathbf{k}}\right] \\
& +g \sqrt{n_{0}} \sum_{\mathbf{k}}\left(d_{\mathbf{k}}^{\dagger} c_{\mathbf{k}}+\text { H.c. }\right)+g \sum_{\mathbf{k}, \mathbf{q}}\left(d_{\mathbf{q}}^{\dagger} c_{\mathbf{q}-\mathbf{k}} b_{\mathbf{k}}+\text { H.c. }\right) .
\end{aligned}
$$

Here, $b_{\mathbf{k}}^{\dagger}$ and $c_{\mathbf{k}}^{\dagger}$ are the creation operators of a boson and the impurity, respectively, with momentum $\mathbf{k}$ and singleparticle energy $\epsilon_{\mathbf{k}}=\mathbf{k}^{2} / 2 m$, where the mass $m$ of a boson and of the impurity are taken to be equal.

In writing the Hamiltonian (1), we already applied the Bogoliubov approximation for the single-particle excitations of the weakly interacting condensate: The operator $\beta_{\mathbf{k}}^{\dagger}$ creates a Bogoliubov excitation and is related to the bare boson operator $b_{\mathbf{k}}^{\dagger}$ by the transformation

$$
b_{\mathbf{k}}^{\dagger}=u_{\mathbf{k}} \beta_{\mathbf{k}}^{\dagger}-v_{\mathbf{k}} \beta_{-\mathbf{k}} .
$$

The coherence factors are $u_{\mathbf{k}}=\sqrt{\left[\left(\epsilon_{\mathbf{k}}+\mu\right) / E_{\mathbf{k}}+1\right] / 2}$ and $v_{\mathbf{k}}=\sqrt{\left[\left(\epsilon_{\mathbf{k}}+\mu\right) / E_{\mathbf{k}}-1\right] / 2}$, the Bogoliubov dispersion is $E_{\mathbf{k}}=\sqrt{\epsilon_{\mathbf{k}}\left(\epsilon_{\mathbf{k}}+2 \mu\right)}$, and the boson chemical potential is $\mu=4 \pi n_{0} a_{B} / m \equiv 1 /\left(2 m \xi^{2}\right)$, where $\xi$ is the coherence length of the condensate. Note that Eq. (1) is defined with respect to the energy of the $\mathrm{BEC}$ in the absence of the impurity.

The interaction between a boson and the impurity is described by the terms in the second line of Eq. (1), where the first of these arises from explicitly extracting the condensate $k=0$ contribution from the second term. For clarity, we write the interaction part of the Hamiltonian in terms of the original boson operator, but note that we always use Eq. (2) to relate this to a Bogoliubov excitation. Within the two-channel model, a boson and the impurity interact by forming a closed-channel dimer created by $d_{\mathbf{k}}^{\dagger}$, with $\epsilon_{\mathbf{k}}^{d}=\epsilon_{\mathbf{k}} / 2$. The interchannel coupling $g$ and the bare detuning $\nu_{0}$ are chosen such that they reproduce the two-body scattering amplitude in vacuum at relative momentum $k$ :

$$
f(k)=-\frac{1}{a^{-1}-\frac{1}{2} r_{0} k^{2}+i k} .
$$

Carrying out the renormalization procedure with highmomentum cutoff $k_{0}$, one obtains [58,59]

$$
a=\frac{m g^{2}}{4 \pi} \frac{1}{\frac{g^{2} m k_{0}}{2 \pi^{2}}-\nu_{0}}, \quad r_{0}=-\frac{8 \pi}{m^{2} g^{2}},
$$

which relates the physical low-energy observables-the scattering length $a$ and effective range $r_{0} \leq 0$ - to the bare parameters of the model. In all calculations, we take the limit $k_{0}, \nu_{0} \rightarrow \infty$ for a given set of values for the observables. We emphasize that our two-channel model describes the exact impurity-boson interaction without any approximations arising from the presence of the condensate. It thus goes beyond the Fröhlich model, which ignores terms where both incoming and outgoing bosons in a scattering process are Bogoliubov excitations. Such terms have been demonstrated to be important beyond the weakcoupling regime [31]. In our model, we recover the singlechannel model (where $r_{0}=0$ and the interaction part of the Hamiltonian has the form $\left.\sum_{\mathbf{k}, \mathbf{k}^{\prime}, \mathbf{q}} b_{\mathbf{k}^{\prime}}^{\dagger} c_{\mathbf{q}-\mathbf{k}^{\prime}}^{\dagger} c_{\mathbf{q}-\mathbf{k}} b_{\mathbf{k}}\right)$ by taking $g \rightarrow \infty$ while keeping $a$ fixed.

To explore the ground-state properties of the unitary Bose polaron, we apply the variational principle using a wave function consisting of terms $\left|\psi_{N}\right\rangle$ with an increasing number $N$ of Bogoliubov modes excited from the condensate by the impurity:

$$
|\Psi\rangle=\left|\psi_{0}\right\rangle+\left|\psi_{1}\right\rangle+\left|\psi_{2}\right\rangle+\left|\psi_{3}\right\rangle .
$$

Explicitly, we have

$$
\begin{aligned}
\left|\psi_{0}\right\rangle= & \alpha_{0} c_{0}^{\dagger}|\Phi\rangle, \\
\left|\psi_{1}\right\rangle= & \left(\sum_{\mathbf{k}} \alpha_{\mathbf{k}} c_{-\mathbf{k}}^{\dagger} \beta_{\mathbf{k}}^{\dagger}+\gamma_{0} d_{0}^{\dagger}\right)|\Phi\rangle, \\
\left|\psi_{2}\right\rangle= & \left(\frac{1}{2} \sum_{\mathbf{k}_{1} \mathbf{k}_{2}} \alpha_{\mathbf{k}_{1} \mathbf{k}_{2}} c_{-\mathbf{k}_{1}-\mathbf{k}_{2}}^{\dagger} \beta_{\mathbf{k}_{1}}^{\dagger} \beta_{\mathbf{k}_{2}}^{\dagger}+\sum_{\mathbf{k}} \gamma_{\mathbf{k}} d_{-\mathbf{k}}^{\dagger} \beta_{\mathbf{k}}^{\dagger}\right)|\Phi\rangle, \\
\left|\psi_{3}\right\rangle= & \left(\frac{1}{6} \sum_{\mathbf{k}_{1} \mathbf{k}_{2} \mathbf{k}_{3}} \alpha_{\mathbf{k}_{1} \mathbf{k}_{2} \mathbf{k}_{3}} c_{-\mathbf{k}_{1}-\mathbf{k}_{2}-\mathbf{k}_{3}}^{\dagger} \beta_{\mathbf{k}_{1}}^{\dagger} \beta_{\mathbf{k}_{2}}^{\dagger} \beta_{\mathbf{k}_{3}}^{\dagger}\right. \\
& \left.+\frac{1}{2} \sum_{\mathbf{k}_{1} \mathbf{k}_{2}} \gamma_{\mathbf{k}_{1} \mathbf{k}_{2}} d_{-\mathbf{k}_{1}-\mathbf{k}_{2}}^{\dagger} \beta_{\mathbf{k}_{1}}^{\dagger} \beta_{\mathbf{k}_{2}}^{\dagger}\right)|\Phi\rangle,
\end{aligned}
$$

with $|\Phi\rangle$ the ground-state wave function of the weakly interacting BEC. Including up to three excitations allows us to investigate the effect of both Efimov trimers and tetramers on the unitary Bose polaron. This goes beyond previous works on the Bose polaron: The effect of dressing the impurity by a single excitation was investigated in Ref. [28] using a $T$-matrix approach and in Ref. [29] with a 
variational approach. Furthermore, two of us previously used two-excitation dressing to investigate the relationship between polaronic and Efimov physics [32], as well as to successfully compare with the experimentally obtained polaron spectral function [16] (see also Ref. [60]).

To investigate the properties of the ground-state Bose polaron, we determine the variational parameters by the stationary condition $\partial_{\alpha^{*}, \gamma^{*}}\langle\Psi|(\hat{H}-E)| \Psi\rangle=0$, where the energy $E$ can be viewed as the Lagrange multiplier that ensures normalization. This yields a set of coupled equations, from which we can eliminate the $\alpha$ coefficients and obtain coupled integral equations for the $\gamma$ 's only. The resulting equations are given in Appendix A. In the $r_{0}$ model, we keep the effective range $r_{0}$ in the two-body interaction, while in the $\Lambda$ model, we set $r_{0}=0$ and apply the cutoff $\Lambda$ to the momenta in the $\gamma$ coefficients.

\section{UNIVERSAL FEW-BODY EFIMOV STATES}

Before proceeding to the many-body polaron physics, it is important to first discuss the few-body limit since-as we explicitly demonstrate in Sec. IV-this plays a crucial role in understanding the polaron properties. To determine the few-body spectrum, we use wave functions of the form (6) in the limit $n \rightarrow 0$, such that $|\Phi\rangle$ now corresponds to the Fock vacuum. Here, the state $\left|\psi_{N}\right\rangle$ with $N$ excitations is identified as a few-body state containing $N$ bosons and the impurity. Consequently, the variational approach is exact and the sectors of different particle numbers completely decouple. Ignoring the trivial equation arising from the single-particle problem, the resulting few-body equations take the form

$$
\begin{gathered}
\mathcal{T}_{0}^{-1}(E, \mathbf{0})=0, \\
\mathcal{T}_{0}^{-1}\left(E-\epsilon_{\mathbf{k}}, \mathbf{k}\right) \gamma_{\mathbf{k}}=\sum_{\mathbf{q}} \frac{\gamma_{\mathbf{q}}}{E-\epsilon_{\mathbf{k q}}}, \\
\mathcal{T}_{0}^{-1}\left(E-\epsilon_{\mathbf{k}_{1}}-\epsilon_{\mathbf{k}_{2}}, \mathbf{k}_{1}+\mathbf{k}_{2}\right) \gamma_{\mathbf{k}_{1} \mathbf{k}_{2}}=\sum_{\mathbf{q}} \frac{\gamma_{\mathbf{k}_{1} \mathbf{q}}+\gamma_{\mathbf{q} \mathbf{k}_{2}}}{E-\epsilon_{\mathbf{k}_{1} \mathbf{k}_{2} \mathbf{q}}},
\end{gathered}
$$

which may be obtained as the zero-density limit of the polaron equations in Appendix A. Here, we have defined $\epsilon_{\mathbf{k}_{1} \mathbf{k}_{2}} \equiv \epsilon_{\mathbf{k}_{1}}+\epsilon_{\mathbf{k}_{2}}+\epsilon_{\mathbf{k}_{1}+\mathbf{k}_{2}}$ and $\epsilon_{\mathbf{k}_{1} \mathbf{k}_{2} \mathbf{q}} \equiv \epsilon_{\mathbf{k}_{1}}+\epsilon_{\mathbf{k}_{2}}+\epsilon_{\mathbf{q}}+$ $\epsilon_{\mathbf{k}_{1}+\mathbf{k}_{2}+\mathbf{q}}$, while $\mathcal{T}_{0}$ is the vacuum two-body $T$ matrix,

$\mathcal{T}_{0}^{-1}(E, \mathbf{k})=\frac{m}{4 \pi a}-\frac{m^{2} r_{0}}{8 \pi}\left(E-\epsilon_{\mathbf{k}}^{d}\right)-\frac{m^{\frac{3}{2}}}{4 \pi} \sqrt{\epsilon_{\mathbf{k}}^{d}-E-i 0}$.

Equation (7) gives the condition for a two-body bound state, while the solutions of Eqs. (8) and (9) yield, respectively, the trimer spectrum and associated tetramers [61]. Note that boson-boson interactions are neglected in this limit since they are only included via a densitydependent mean-field shift in the boson chemical potential.
However, this approximation is reasonable when the Efimov states are insensitive to $a_{B}$, i.e., when the microscopic length scale determining the three-body parameter greatly exceeds $a_{B}$.

In Fig. 1, we show the spectrum of bound states obtained by solving Eqs. (7)-(9) within the $r_{0}$ model. For a positive scattering length, a single two-body bound dimer exists, with energy

$$
\varepsilon_{B}=-\frac{\left(\sqrt{1-2 r_{0} / a}-1\right)^{2}}{m r_{0}^{2}},
$$

as found by solving Eq. (7). The three-body problem described by Eq. (8) is distinctly different: For strong interactions, the impurity and two bosons can form an infinite series of three-body bound states, even in the absence of the two-body bound state. Remarkably, these so-called Efimov trimers [21,62] satisfy a discrete scaling symmetry whereby the energy $E$ of one trimer can be mapped onto the next via the discrete transformation $E \rightarrow \lambda_{0}^{-2 l} E$ and $a \rightarrow \lambda_{0}^{l} a$, with $l$ an integer and $\lambda_{0}$ a scaling parameter that depends on the particular three-body problem (for reviews, see Refs. [22,24-26]). In our case of two noninteracting identical bosons with the same mass as the third particle, the scaling factor is found from a transcendental equation to be $\lambda_{0}=e^{\pi / s_{0}} \simeq 1986.1$ [62]. Because of this large scaling factor, we only show the two lowest trimer states in the figure (indeed, for realistic experimental parameters, the size of the first excited Efimov trimer is of order $1 \mathrm{~cm}$ ).

Below the ground-state Efimov trimer, we predict the existence of two tetramer states, even at unitarity (see Fig. 1). This mirrors previous results for four identical bosons, where it was found, both theoretically [63-66] and experimentally [67], that there are two lower-lying tetramers associated with the ground-state trimer. The present scenario more closely resembles studies of a light atom resonantly interacting with three identical heavy atoms $[47,68,69]$. Our findings are generally consistent with the results of these previous works, although no excited tetramer was reported for small mass imbalance [70]. Note that, within our theory, we do not require an additional length scale to fix the positions of the tetramers relative to the Efimov trimers [65].

A crucial point is that the few-body physics described here is essentially universal, in the sense that the entire spectrum is independent of the details of the short-range physics. This universality originates from the large separation of scales between the short-distance physics, characterized here by the effective range $r_{0}$, and the typical size of the ground-state Efimov trimer. The latter may be conveniently characterized by the "critical" scattering length $a_{-}$at which the trimer crosses into the three-atom continuum. In our model, we have $a_{-} / r_{0} \simeq 2467$ [72] (see Table I for a summary of the length scales and energy scales associated 
TABLE I. Comparison of few-body data for the $r_{0}$ and the $\Lambda$ models. While the overall length scale set by $a_{-}$depends on the details of the models, the dimensionless ratios characterizing the few-body spectra agree to an accuracy of $1 \%$ or less. These quantities include the scattering lengths at which the few-body bound states cross into the continuum, as well as their energies at unitarity, and are illustrated in Fig. 1.

\begin{tabular}{|c|c|c|c|c|c|c|c|c|}
\hline & $\left|a_{-}\right|$ & $m a_{-}^{2}\left|E_{-}\right|$ & $a_{-}^{(1)} / a_{-}$ & $\sqrt{E_{-} / E_{-}^{(1)}}$ & $a_{-} / a_{4 B,-}^{(1)}$ & $E_{4 B,-}^{(1)} / E_{-}$ & $a_{-} / a_{4 B,-}^{(2)}$ & $E_{4 B,-}^{(2)} / E_{-}$ \\
\hline$r_{0}$ model & $2467\left|r_{0}\right|$ & 9.913 & 1991 & 1986.126 & 2.810 & 9.35 & 1.060 & $1.0030(3)$ \\
\hline$\Lambda$ model & $1354 \Lambda^{-1}$ & 9.950 & 1987 & 1986.127 & 2.814 & 9.43 & 1.061 & $1.0036(1)$ \\
\hline
\end{tabular}

with Efimov physics). This large ratio ensures that the energy of the ground-state Efimov trimer at unitarity is far smaller than that required to probe the short-range physics. Indeed, we see that the ratio of critical scattering lengths for the excited- and ground-state trimers, i.e., $a_{-}^{(1)} / a_{-} \simeq 1991$, is very close to the predicted scaling factor of 1986.1. Likewise, at unitarity, the ratio of the ground-state trimer energy to that of the excited state is $E_{-} / E_{-}^{(1)} \simeq(1986.1)^{2}$; i.e., it matches the universal prediction to 5 significant digits. This is in remarkable contrast to the situation for three identical bosons, where deviations from the universal scaling factor of 22.7 are usually on the order of $10 \%-20 \%$ because of finite-range corrections [22,24,73].

To test the model independence of the few-body physics, we also consider the $\Lambda$ model, where $r_{0}=0$ and we apply an explicit cutoff $\Lambda$ to the momentum sums in the three- and four-body equations (8) and (9). Here, we find that after fixing the three-body parameter, the spectrum is essentially indistinguishable from that in Fig. 1, in the sense that the differences are within the thickness of the lines [74]. The agreement between the two models is also evident in the summary of length scales and energy scales in Table I. Thus, we conclude that our description of the fewbody spectrum can be accurately characterized by universal Efimov theory.

Further evidence of the universality of our few-body results is found when comparing the ratio of our groundstate tetramer and trimer energies with recent QMC results [33]. There, this ratio was reported to be $E_{4 B,-}^{(1)} / E_{-} \simeq 9.7$, while we find $E_{4 B,-}^{(1)} / E_{-} \simeq 9.35$ and 9.43 , respectively, for the $r_{0}$ and $\Lambda$ models (Table I). Importantly, the QMC calculation used a completely different model involving a hard-sphere boson-boson interaction, characterized by the scattering length $a_{B}$, and attractive square-well interactions between the impurity and the bosons. The range of the square well was taken to be much smaller than $a_{B}$, and consequently, the energies of the ground-state Efimov trimer and associated tetramers are set by $a_{B}$. The universality of the spectrum thus means that we can relate the three-body parameter in the QMC calculation to the bosonboson scattering length [76]:

$$
a_{-}^{(\mathrm{QMC})} \simeq-2.09(36) \times 10^{4} a_{B}^{(\mathrm{QMC})} .
$$

The agreement between the results from the different models also reinforces the point that we do not need a four-body parameter to fix the tetramer relative to the trimer. This is consistent with theoretical results for identical bosons $[63,64,66]$ and for heteronuclear systems [47,68,69].

We end this section by contrasting the few-body universality found here with the so-called van der Waals universality of Efimov physics [77-81]. The latter refers to the phenomenon where the Efimov states of various atomic species are universally related to their van der Waals length, e.g., $a_{-} \simeq-(9.1 \pm 1.5) r_{\mathrm{vdW}}$. This behavior, however, is rather different from that predicted by universal zero-range Efimov theory, in particular, for the ground-state trimer. Indeed, the ratio $a_{-}^{(1)} / a_{-} \simeq 20.7$ found in van der Waals universal theory [81] significantly deviates from the universal ratio 22.7 , in contrast to the scenario considered here.

\section{POLARON ENERGY AT UNITARITY}

We now turn to the many-body system and investigate the zero-temperature equation of state for the polaron at unitarity. Since we wish to properly describe Efimov physics within our models, we focus on the regime where the boson-boson scattering length $a_{B}$ is always much smaller than all other length scales. To test the accuracy of our variational approach outlined in Eqs. (5) and (6), we consider wave functions with up to one, two, or three Bogoliubov excitations, corresponding to $\left|\psi_{0}\right\rangle+\left|\psi_{1}\right\rangle$, $\left|\psi_{0}\right\rangle+\left|\psi_{1}\right\rangle+\left|\psi_{2}\right\rangle$, and $|\Psi\rangle$, respectively. For each model, we convert the short-distance scale (e.g., $r_{0}$ or $\Lambda^{-1}$ ) into the dimensionless three-body parameter $n^{1 / 3}\left|a_{-}\right|$in order to expose the role of universal Efimov physics. The numerical results are displayed in Fig. 2, and, in the following, we analyze the different regimes defined by particle density.

\section{A. Low-density limit}

In the limit of vanishing density $n^{1 / 3}\left|a_{-}\right| \rightarrow 0$, the groundstate polaron energy should reduce to that of the deepest bound few-body cluster. If the polaron variational wave function only includes one Bogoliubov excitation, the largest few-body state it can describe is the dimer, which has zero binding energy at unitarity. Thus, as shown in Fig. 2, we obtain an energy that simply tends to zero with decreasing density, i.e., $E=-(4 \pi n)^{2 / 3} / m \simeq-5.4 n^{2 / 3} / m$ 


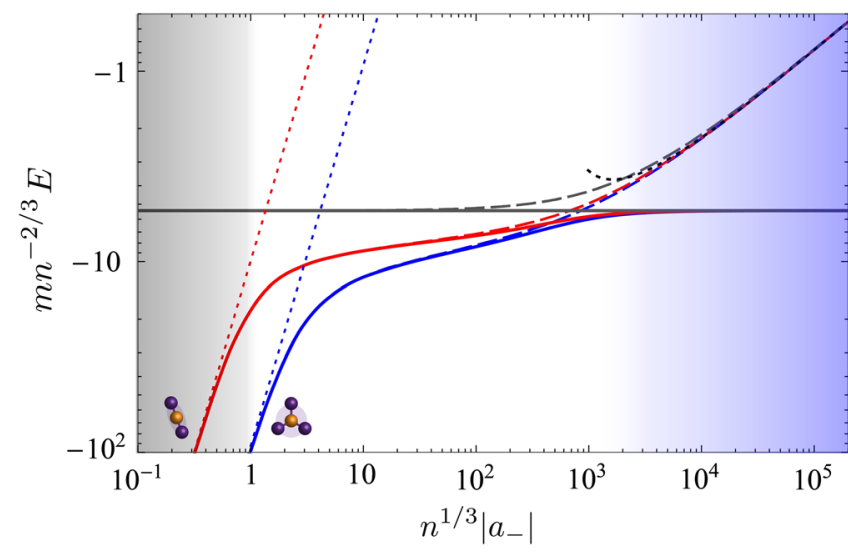

FIG. 2. Energy equation of state for the Bose polaron at unitarity $1 / a=0$, obtained using the variational wave function with one (gray lines), two (red lines), and three (blue lines) Bogoliubov excitations for vanishing boson-boson interactions $a_{B} \rightarrow 0$. We show the results of the $r_{0}$ model (dashed lines) and the $\Lambda$ model (solid lines). In the low-density limit, the dotted straight lines correspond to the Efimov trimer and associated tetramer energies, with $E_{-}=-9.91 / m\left|a_{-}\right|^{2}$ and $E_{4 B,-}^{(1)}=-92.7 / m\left|a_{-}\right|^{2}$, respectively. The black dotted line in the high-density regime shows the result of a perturbative expansion, Eq. (14). The gray shaded region depicts the low-density few-body dominated regime, while the blue shaded region corresponds to the high-density regime $\left|r_{0}\right|, \Lambda^{-1}>n^{-1 / 3}$, where Efimov physics is suppressed and the system is sensitive to the microscopic model.

$[28,29]$. Note that this is independent of the microscopic model we use since the interparticle spacing greatly exceeds any length scale of the impurity-boson interactions.

For polaron wave functions with up to two (three) Bogoliubov excitations, the low-density limit recovers the deepest bound universal trimer (tetramer) state discussed in Sec. III. Here, the density drops out of the problem, and we have polaron energy $E=-\eta /\left(m\left|a_{-}\right|^{2}\right)$, where $\eta$ is a universal, model-independent constant that depends on the Efimov cluster size (see Fig. 2).

We also expect there to be larger bound clusters beyond the tetramer, similar to the case of identical bosons where larger clusters associated with the Efimov effect have been predicted [82-84] and, indeed, experimentally observed [85]. For the impurity case, recent QMC calculations already demonstrate the existence of a pentamer with $\eta \simeq$ 300 [33]. However, since there is a high-energy cutoff in the problem (e.g., set by $\Lambda$ or $r_{0}$ ), the system cannot support arbitrarily deeply bound Efimov clusters, and the deepest bound state should be universal as long as $\eta \ll 10^{6}$. Indeed, as suggested by the QMC calculations [33], it is possible that there is no bound cluster larger than the pentamer for the equal-mass impurity case. First, the pairwise attraction between the impurity and the bosons scales with $N$ rather than $N(N-1)$, unlike the case for $N$ identical bosons [25], so larger bound states will be less favored. Second, an effective short-range repulsion between bosons could mean that the pentamer has a closed-shell structure, where any additional bosons must occupy higher-energy orbitals, similar to what has been argued for mass-imbalanced fermions [86,87]. Such an effective repulsion naturally emerges in both the $r_{0}$ and the $\Lambda$ models: In the former, this arises from the fact that only one boson at a time can occupy the closed-channel dimer, while in the latter, it is due to the three-body cutoff being equivalent to a threebody repulsion [56].

From the above considerations, it is reasonable to conclude that the low-density limit is universal. Moreover, since there is a maximum size to the deepest bound Efimov cluster, we expect the polaron energy to have a well-defined thermodynamic limit, where $m E / n^{2 / 3}$ is finite for nonzero density.

\section{B. High-density limit}

To further support the claim that the polaron energy is finite at unitarity, we consider the opposite limit of high density, $n \rightarrow \infty$. Here, the interparticle spacing becomes smaller than the range of the interactions, and thus, the system will depend on the microscopic details of the model. However, in the case of the $r_{0}$ model, it allows us to perform a controlled perturbative expansion in the interchannel coupling $g$ (or, equivalently, $1 / n^{1 / 3}\left|r_{0}\right|$ ) at unitarity [88]. Physically, this corresponds to the limit of a narrow Feshbach resonance, a scenario that has already been successfully investigated for an impurity in a Fermi sea, both theoretically [89-91] and experimentally [92,93].

Our starting point is the self-energy of the unitary Bose polaron, which, in the limit $g \rightarrow 0$ and $a_{B} \rightarrow 0$, consists of the lowest-order diagrams shown in Fig. 3. Such diagrams are included in the self-consistent $T$-matrix approach to the Bose polaron [28]. For an impurity with momentum $\mathbf{p}$ and frequency $\omega$, the self-energy in this limit is explicitly (a)

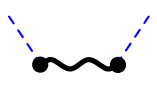

(c)

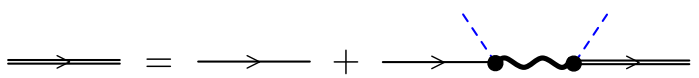

FIG. 3. Lowest-order diagrams for the unitary ground-state polaron in the high-density limit $n^{1 / 3}\left|r_{0}\right| \gg 1$, where (a) and (b) give, respectively, the first- and second-order terms for the polaron energy in a weakly interacting BEC. Here, the dashed lines correspond to particles emitted or absorbed from the condensate, the blue solid line is the boson propagator, the wavy lines represent closed-channel molecule propagators, and the filled circles correspond to the interchannel coupling $g$. The black double line, defined in (c), represents the dressed impurity to lowest order, where the black single line is the bare impurity propagator. 


$$
\begin{aligned}
\Sigma(\mathbf{p}, \omega) \simeq & \frac{n_{0} g^{2}}{\omega-\epsilon_{\mathbf{p}}^{d}}+\frac{n_{0} g^{4}}{\left(\omega-\epsilon_{\mathbf{p}}^{d}\right)^{2}} \\
& \times \sum_{\mathbf{k}}\left[\frac{1}{\omega-\epsilon_{\mathbf{k}+\mathbf{p}}-\epsilon_{\mathbf{k}}-\frac{n_{0} g^{2}}{\omega-\epsilon_{\mathbf{k}+\mathbf{p}}^{d}-\epsilon_{\mathbf{k}}}}+\frac{1}{2 \epsilon_{\mathbf{k}}}\right],
\end{aligned}
$$

where the first and second terms correspond to the diagrams in Figs. 3(a) and 3(b), respectively. The ground-state polaron energy is then determined by taking the zero-momentum pole of the impurity propagator, which gives $E=\Sigma(\mathbf{0}, E)$. Note that we must include self-energy insertions in the impurity propagator even at lowest order (see Fig. 3) since we cannot simply take $\omega=0$ at the pole when $a_{B} \rightarrow 0$. This is in contrast to previous perturbative treatments of the weakly interacting Bose polaron which focused on $a_{B}>0$ [31].

In the regime of weak boson-boson interactions, where $n a_{B}^{3} \ll a_{B} /\left|r_{0}\right| \ll 1$, we obtain the ground-state polaron energy

$$
E \simeq-\frac{1}{m} \sqrt{\frac{8 \pi n}{\left|r_{0}\right|}}+\frac{1}{m} \sqrt{\frac{3}{7}}\left(\frac{8 \pi n}{\left|r_{0}\right|^{5}}\right)^{1 / 4}
$$

This demonstrates that the energy is well defined and bounded from below in this limit, even when the bosonboson interactions are vanishingly small. Such behavior arises from the fact that only one boson at a time can scatter into the closed channel, thus producing an effective bosonboson repulsion that restricts the density of bosons that can cluster around the impurity. As shown in Fig. 2, the perturbative expression correctly reproduces the energy for the $r_{0}$ model in the high-density limit. Note that the oneexcitation wave function only captures the leading-order term in Fig. 3(a), while the wave functions with two or three excitations correctly describe the next order correction in $1 / n^{1 / 3}\left|r_{0}\right|$.

In the case of the $\Lambda$ model, the high-density limit corresponds to $n^{1 / 3} / \Lambda \rightarrow \infty$, which is equivalent to taking the cutoff $\Lambda$ to zero. Therefore, within our variational approach, only two-body correlations survive, and we obtain the equations for the wave function with one excitation (see Appendix A). For vanishing boson-boson interactions, this yields $E=-(4 \pi n)^{2 / 3} / m$, which is the polaron energy of the one-excitation wave function across all densities, as shown in Fig. 2.

\section{Many-body universal regime}

In the intermediate regime $r \ll n^{-1 / 3} \ll\left|a_{-}\right|$(where $r$ can represent $\left|r_{0}\right|$ or $\Lambda^{-1}$ ), the interparticle distance is well separated from all length scales associated with the interactions. If the medium were fermionic rather than bosonic, then the polaron energy at unitarity, in the limit $r \rightarrow 0$, would be a universal value that only depends on the medium density, i.e., $E_{\mathrm{pol}} \simeq-4.6 n^{2 / 3} / m$ [48]. Remarkably, for the Bose polaron, we see in Fig. 2 that the energy strongly depends on $n^{1 / 3}\left|a_{-}\right|$at intermediate densities. This suggests that there exist resonant three-body interactions, such that there are strong three-body correlations in the system even when the trimer binding energy is comparatively small.

Even though the energy of the Bose polaron cannot be assigned a universal value at unitarity, we argue that it is, in fact, a universal function of $n^{1 / 3}\left|a_{-}\right|$away from the highdensity regime. We have previously argued that the lowdensity limit of the polaron energy universally depends on $n^{1 / 3}\left|a_{-}\right|$because of the universal behavior of the few-body states demonstrated in Sec. III. In Fig. 2, we clearly see that this universality extends to the intermediate-density regime. Moreover, even for the higher densities shown in Fig. 4, we see that different microscopic models of the Bose polaron can essentially be collapsed onto the same curve when the ground-state energy is plotted versus $n^{1 / 3}\left|a_{-}\right|$. Note that the small difference between the curves of the $\Lambda$ and the $r_{0}$ models is due to $n^{1 / 3}\left|r_{0}\right|$ corrections to the two-body scattering properties. Such a deviation from universality would also be present in the unitary Fermi system at these densities [89-91].

Crucially, both the results for the $\Lambda$ and the $r_{0}$ models are consistent with the QMC calculation from Ref. [36] when Eq. (12) is used to plot the QMC data in terms of the Efimov three-body parameter. This demonstrates that the polaron energy can be universally described in terms of $n_{0}^{1 / 3}\left|a_{-}\right|$, regardless of the microscopic details. Furthermore, it suggests that our variational approach with three Bogoliubov excitations captures the dominant correlations in the full many-body problem, and that the Efimov

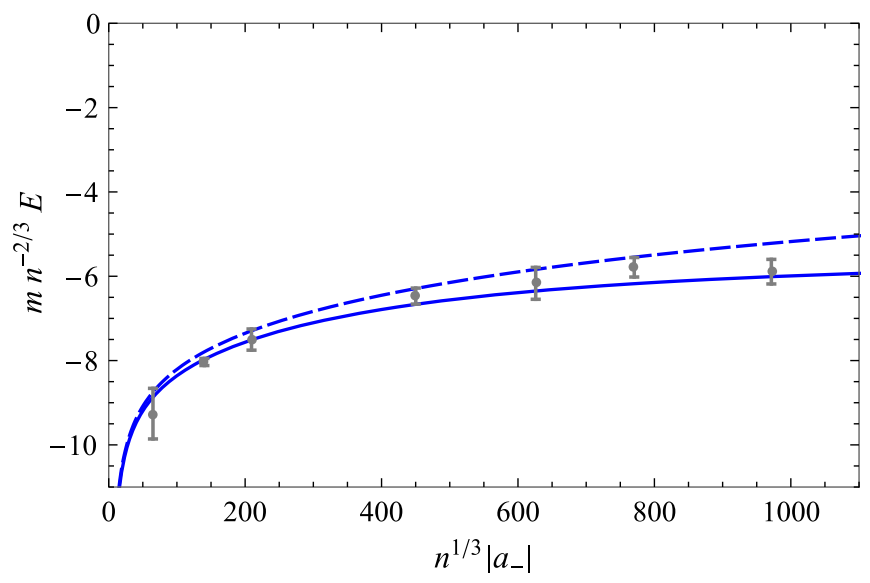

FIG. 4. Comparison of different models for the unitary Bose polaron in the regime $r \ll n^{-1 / 3} \ll\left|a_{-}\right|$, where $r$ is the range of the interactions. We show the results for the polaron energy from the $\Lambda$ model (solid line) and the $r_{0}$ model (dashed line), which include up to three excitations of the condensate and which take $a_{B} \rightarrow 0$. The QMC results of Ref. [36] are shown as the gray dots. The recent Aarhus experiment [16] is estimated to have $n^{1 / 3}\left|a_{-}\right| \simeq 100$. 
three-body parameter plays a more important role in the polaron energy than the coherence length of the condensate (see Appendix B). We emphasize that our comparison in Fig. 4 constitutes a complete reinterpretation of the QMC results of Ref. [36] since that work did not consider the possibility that the variation of the polaron energy with boson-boson interaction was due to Efimov physics.

\section{CHARACTERIZATION OF THE POLARON}

Having shown that the polaron energy is a universal function of $n^{1 / 3}\left|a_{-}\right|$for sufficiently low densities, we now turn to the other quasiparticle properties that characterize the polaron: the residue, effective mass, and contact. We generally focus on the $r_{0}$ model, which is the more physical model and which allows a perturbative analysis in the highdensity limit.

\section{A. Residue}

The residue $Z$ is the squared overlap of the polaron wave function with the noninteracting state $c_{0}^{\dagger}|\Phi\rangle$. Making reference to the wave function in Eq. (6), this is

$$
Z=\left|\alpha_{0}\right|^{2}
$$

where we assume $\langle\Psi \mid \Psi\rangle=1$ for normalization (see Appendix C). The residue can be accessed directly in experiment; indeed, in the case of the Fermi polaron, it has been measured using both radio-frequency spectroscopy [94] and Rabi oscillations [92].

In Fig. 5 we show our results for the polaron residue at unitarity as a function of the three-body parameter, where the boson-boson interactions are taken to be vanishingly

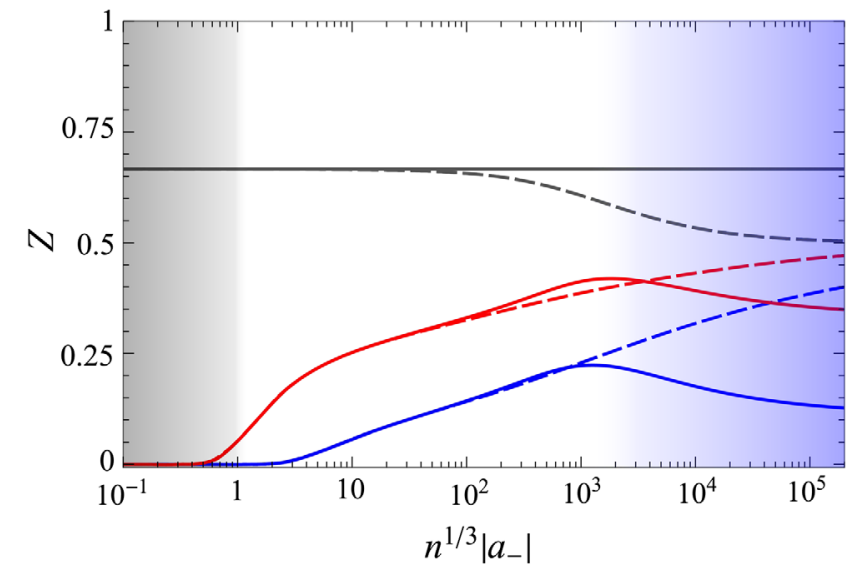

FIG. 5. Polaron residue $Z$ at unitarity $1 / a=0$ as a function of the dimensionless three-body parameter. We show the results for the $\Lambda$ model (solid line) and the $r_{0}$ model (dashed line) for $a_{B} \rightarrow 0$. In order of decreasing residue, the results are obtained for wave functions including up to one, two, or three excitations of the condensate. The grey and blue shaded regions are the same as in Fig. 2, i.e., the low- and high-density regimes, respectively. small. We find that there are clear differences between the results depending on the number of Bogoliubov excitations used in the variational approach, and also between the different models. Within the $r_{0}$ model, the residue takes the value $1 / 2$ in the high-density regime where the polaron wave function is an equal superposition of the impurity and the closed-channel dimer. However, while the residue within the one-excitation approximation is seen to increase from $1 / 2$ to $2 / 3$ with increasing density, we observe a rapid decrease of $Z$ towards the low-density regime when including several excitations. On the other hand, for the $\Lambda$ model, the residue is $2 / 3$ if we consider only one Bogoliubov excitation. By increasing the number of Bogoliubov excitations, it is suppressed not only in the few-body dominated regime but also in the high-density limit, where the residue takes the values $1 / 3$ and $1 / 9$, respectively, when two and three excitations are taken into account. Thus, unlike the energy, the polaron residue does not appear to converge to a universal function of the threebody parameter.

The origin of this behavior can be understood within perturbation theory for the $r_{0}$ model in the high-density limit. Considering again the diagrams in Fig. 3 and the explicit expression for the self-energy in Eq. (13), we find, at small $g$,

$$
\begin{aligned}
Z^{-1} & =1-\left.\frac{\partial \Sigma(\mathbf{0}, \omega)}{\partial \omega}\right|_{\omega=E} \\
& \simeq 2-\sqrt{\frac{3}{7}} \frac{2}{\left(8 \pi n\left|r_{0}\right|^{3}\right)^{1 / 4}}+\frac{L}{\left|r_{0}\right|},
\end{aligned}
$$

where $L$ is a low-energy length scale that provides an infrared cutoff of low momenta. In the thermodynamic limit, this is set by the coherence length of the BEC; i.e., we have $L \propto \xi$. Thus, we find that the residue vanishes when the coherence length $\xi \rightarrow \infty$, and consequently, the polaron wave function is orthogonal to the noninteracting impurity state in the case of an ideal BEC. This feature cannot properly be captured within our variational approach since this requires an infinite number of Bogoliubov excitations, hence the observed lack of convergence in Fig. 5. On the other hand, when $a_{B} \neq 0$, we find that the wave functions with two and three excitations both give the same leadingorder correction to $Z$ :

$$
Z-\frac{1}{2} \propto \sqrt{\frac{a_{B}}{\left|r_{0}\right|}}
$$

in the limit $n^{1 / 3} a_{B} \ll 1$ and $n^{1 / 3}\left|r_{0}\right| \rightarrow \infty$ [95]. Thus, in this case, the residue is finite and initially increases with decreasing $\left|r_{0}\right|$. Note, however, that the residue always vanishes in the low-density regime, as shown in Fig. 5, since the polaron evolves into a few-body bound state which has zero overlap with the noninteracting impurity state. 
The simultaneous convergence of the energy and suppression of the residue in the ideal BEC arises from the fact that the impurity affects the condensate at arbitrarily large distances and thus excites an infinite number of low-energy modes in the condensate. This result is akin to the orthogonality catastrophe for a static impurity in a Fermi gas. Indeed, in that case, the orthogonality of the interacting and noninteracting impurity wave functions is also apparent in perturbation theory, already at lowest order beyond mean-field theory [96]. We also note that the vanishing residue of the Bose polaron is not confined to the strongly interacting impurity at unitarity: Even for weak impurityboson interactions, the residue of the attractive polaron was found within perturbation theory to approach zero as $1 / \xi$ when $a_{B} \rightarrow 0$ [31].

\section{B. Effective mass}

The interactions between the impurity and the bosonic medium also modify the mobility of the impurity and give rise to an effective mass $m^{*}$. This can be derived from the impurity's self-energy as follows:

$$
\frac{m}{m^{*}}=Z\left[1+\left.m \frac{\partial^{2} \Sigma(\mathbf{q}, E)}{\partial q^{2}}\right|_{q=0}\right]
$$

Note that this gives $m^{*}=m$ for the noninteracting impurity state, as required. Our calculation of $m^{*}$ within the variational approach is described in Appendix $C$, and the results for $a_{B} \rightarrow 0$ at unitarity are plotted in Fig. 6. Because of the complexity of the calculation, we restrict ourselves to wave functions with up to two Bogoliubov excitations.

For the case of an ideal BEC, we have residue $Z \rightarrow 0$, and thus one might conclude from Eq. (18) that the effective mass diverges. However, if we once again

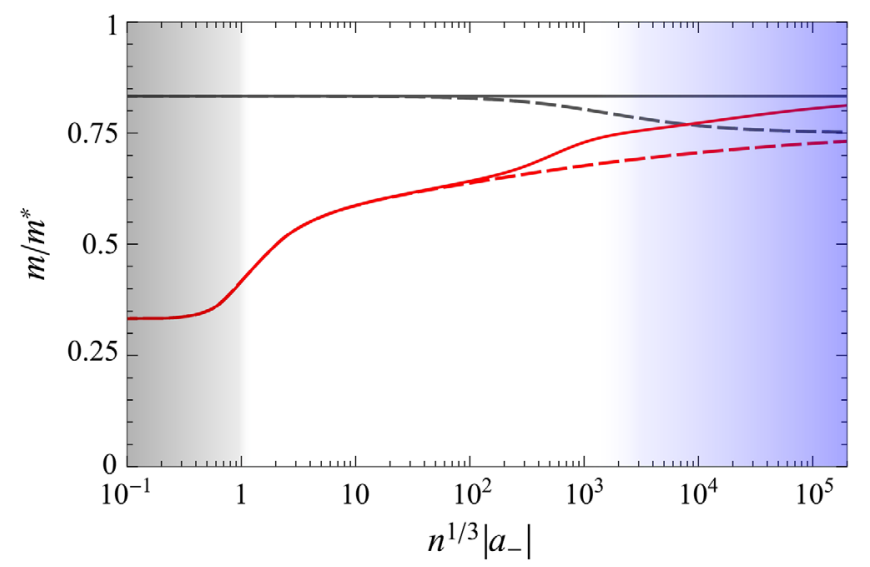

FIG. 6. Inverse effective mass of the Bose polaron at unitarity, $1 / a=0$, for $a_{B}=0$. We calculate it with the $\Lambda$ model (solid lines) and the $r_{0}$ model (dashed lines), taking into account one (gray lines) and two (red lines) Bogoliubov excitations. The grey and blue shaded regions are the same as in Fig. 2. consider the high-density limit of the $r_{0}$ model and use the self-energy in Eq. (13), we obtain

$$
\frac{m}{m^{*}} \simeq Z\left(\frac{3}{2}-\sqrt{\frac{3}{7}} \frac{1}{\left(8 \pi n\left|r_{0}\right|^{3}\right)^{1 / 4}}+\alpha \frac{L}{\left|r_{0}\right|}\right),
$$

where $\alpha$ is a constant that must be determined from a proper finite- $a_{B}$ perturbative analysis. Thus, we see that there is a term proportional to $L$ that cancels the corresponding term in the residue in Eq. (16), implying that the effective mass is finite.

Indeed, in the low-density few-body regime, we already have the situation where the residue vanishes, while the effective mass remains finite with $m^{*}=(N+1) m$, where $N$ is the number of bosons bound to the impurity. This behavior is captured by both $r_{0}$ and $\Lambda$ models, as shown in Fig. 6, where the wave function with two Bogoliubov excitations has $m^{*} / m \rightarrow 3$ as $n \rightarrow 0$. Likewise, we expect the wave function with three excitations to recover the mass of the tetramer in this limit. For higher densities, we find that the results of our variational approach become sensitive to the specific model used, as well as to the number of excitations of the ideal BEC considered. Here, in the manybody limit, one requires an infinite number of low-energy excitations to correctly describe the effective mass for $a_{B} \rightarrow 0$, similarly to the case of the residue.

For a BEC with $0<n^{1 / 3} a_{B} \ll 1$, the behavior of the effective mass in the high-density limit of the $r_{0}$ model can once again be captured using just two Bogoliubov excitations of the BEC. In the limit $\left|r_{0}\right| \rightarrow \infty$, the polaron effective mass $m^{*} / m \rightarrow 4 / 3$, which corresponds to twice the reduced mass of the impurity and closed-channel dimer. Moreover, we find that the leading-order correction behaves as

$$
\frac{m}{m^{*}}-\frac{3}{4} \propto \sqrt{\frac{a_{B}}{\left|r_{0}\right|}}
$$

Thus, the effective mass, in this case, initially decreases with decreasing density before increasing towards the mass of the deepest bound few-body cluster.

\section{Contact}

Another quantity of interest is the unitary Tan contact, which is defined from the polaron energy as $[97,98]$

$$
C=\left.m^{2} g^{2} \frac{\partial E}{\partial \nu}\right|_{\nu=0}
$$

Here, we have used the physical detuning $\nu \equiv-g^{2} m / 4 \pi a$, which can be related to the bare detuning $\nu_{0}$ using Eq. (4). Physically, the contact gives a measure of the density of pairs at short distances. For the two-channel Hamiltonian in 


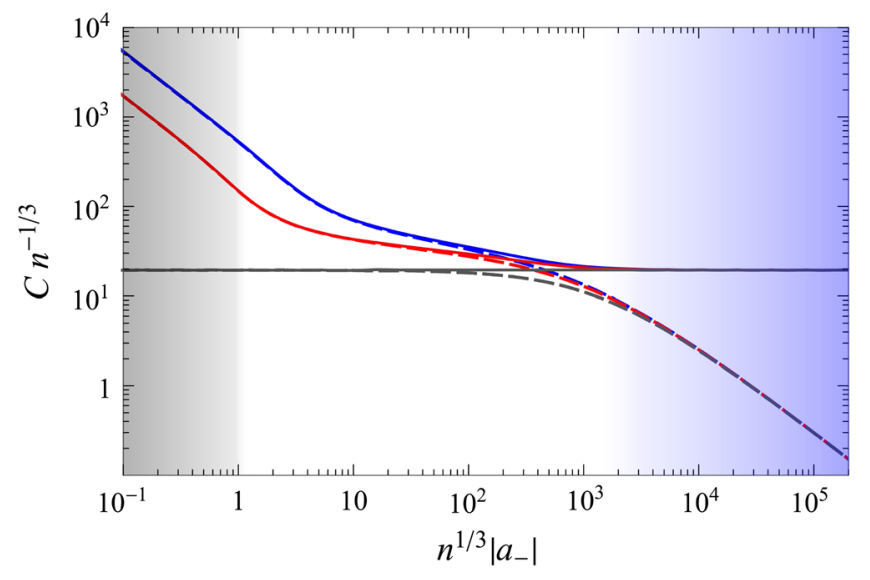

FIG. 7. Contact of the Bose polaron at unitarity, $1 / a=0$, for $a_{B}=0$. The solid and dashed curves correspond to the $\Lambda$ and $r_{0}$ models, respectively. We show the results obtained using wave functions with up to one (gray line), two (red line), and three (blue line) excitations. The grey and blue shading indicates the same regions as in Fig. 2.

Eq. (1), one can show that the contact is proportional to the population of closed-channel dimers [98]:

$$
C=m^{2} g^{2} \sum_{\mathbf{k}}\left\langle\Psi\left|d_{\mathbf{k}}^{\dagger} d_{\mathbf{k}}\right| \Psi\right\rangle .
$$

Within our variational ansatz, this corresponds to

$$
C=m^{2} g^{2}\left(\left|\gamma_{0}\right|^{2}+\sum_{\mathbf{k}}\left|\gamma_{\mathbf{k}}\right|^{2}+\frac{1}{2} \sum_{\mathbf{k}_{1} \mathbf{k}_{2}}\left|\gamma_{\mathbf{k}_{1} \mathbf{k}_{2}}\right|^{2}\right) .
$$

Note that the limit $g \rightarrow \infty$ is well defined, and thus this expression also captures the single-channel $\Lambda$ model.

Figure 7 displays the contact at unitarity for both $\Lambda$ and $r_{0}$ models in the case of vanishing boson-boson interactions. Similarly to the polaron energy, we find that the contact in the high-density limit is well converged with respect to the number of Bogoliubov excitations. For the $r_{0}$ model, we can evaluate the contact perturbatively for large $n^{1 / 3}\left|r_{0}\right|$ using the self-energy in Eq. (13). Here, we simply shift the closed-channel dispersion from $\epsilon_{\mathbf{p}}^{d}$ to $\epsilon_{\mathbf{p}}^{d}+\nu$ and then determine the ground-state energy from the equation $E=\Sigma(\mathbf{0}, E)$ as a function of detuning $\nu$. Applying Eq. (21) then yields the high-density expression

$$
C \simeq \frac{8 \pi}{\left|r_{0}\right|}\left(\frac{1}{2}-\frac{29}{28} \sqrt{\frac{3}{7}} \frac{1}{\left(8 \pi n\left|r_{0}\right|^{3}\right)^{1 / 4}}\right)
$$

We find that this perturbative result is correctly reproduced by both two- and three-excitation approximations in the high-density limit. The leading-order term is independent of density since the closed-channel fraction tends to $1 / 2$ at unitarity as $\left|r_{0}\right| \rightarrow \infty$ (or, equivalently, as $g \rightarrow 0$ ); see Eq. (16).
For lower densities $n^{1 / 3}\left|a_{-}\right| \lesssim 10^{3}$, the contact becomes insensitive to the underlying model and thus appears to be a universal function of the dimensionless three-body parameter (see Fig. 7). In the limit of vanishing density $n \rightarrow 0$, we recover the contact of the deepest bound few-body cluster, where $C\left|a_{-}\right|$is a universal number. For the Efimov trimer, we have $C\left|a_{-}\right| \simeq 171.4$ in the $r_{0}$ model and $C\left|a_{-}\right| \simeq 172.2$ in the $\Lambda$ model, while for the associated tetramer, we obtain $C\left|a_{-}\right| \simeq 535.5$ ( $r_{0}$ model $)$ and 542.6 ( $\Lambda$ model $)$.

\section{CONCLUSIONS AND OUTLOOK}

Using a variational approach, we have investigated the ground state of the Bose polaron with unitarity limited impurity-boson interactions. For sufficiently low boson densities, we have demonstrated that the polaron equation of state is a universal function of the three-body parameter associated with Efimov physics. This is a highly nontrivial result since the regime of universality extends to densities at which the binding energies of the few-body states are several orders of magnitude smaller than the polaron energy. Our findings are corroborated by the fact that we observe the same behavior for two different microscopic models and by the fact that our calculations agree well with recent QMC results $[33,36]$ when they are appropriately reinterpreted in terms of the three-body parameter. We have also demonstrated that the few-body spectrum of Efimov trimers and associated tetramers is model independent, further highlighting the universality of our results.

In the nonuniversal, high-density limit, we have performed a controlled perturbative analysis of the $r_{0}$ model, and we have derived new analytic expressions for the unitary Bose polaron at a narrow Feshbach resonance. Our results demonstrate that even when we take the ideal Bose gas limit, the polaron energy and contact can remain well defined thermodynamically. This is a consequence of the fact that only one boson at a time can occupy the closedchannel dimer state, which thus generates an effective repulsion between bosons that acts to stabilize the system. We expect a similar scenario for the $\Lambda$ model since the high-momentum cutoff in the three- and four-body terms of the variational equations is equivalent to an explicit threebody repulsion [56]. However, in general, it is not clear that a well-defined thermodynamic limit exists for treatments of the Bose polaron that focus on single-channel Hamiltonians and neglect Efimov physics [39,99].

While the polaron equation of state at unitarity is relatively insensitive to boson-boson interactions, we find that the quasiparticle residue and effective mass strongly depend on the coherence length of the BEC. In particular, the residue vanishes as $\xi \rightarrow \infty$, a feature that resembles the orthogonality catastrophe [53]. Thus, an interesting remaining question is how the residue tends to zero with the number of excitations of the condensate included in the wave function. A related question is as follows: What finite 
result does the effective mass converge towards as $\xi \rightarrow \infty$, and is it a universal function of the three-body parameter?

We emphasize that the (two-channel) $r_{0}$ model faithfully describes the physics of an actual Feshbach resonance in an ultracold atomic gas [57], and thus we expect our results to accurately describe current and future cold atom experiments. Furthermore, we expect the universal behavior presented here to also exist for heavy impurities, a scenario that can be realized by choosing atomic species with a large mass ratio or by pinning the impurity in an optical lattice. In the particular case of a static impurity, there are no Efimov few-body states, and therefore, it would be of great interest to understand how the system behaves in the limit $n^{1 / 3}\left|r_{0}\right| \ll 1$ of the two-channel model.

Our results may be probed in experiments similar to those carried out recently at JILA [15] and in Aarhus [16]. In particular, our results directly apply to the latter experiment, which used ${ }^{39} \mathrm{~K}$ as both the impurity and host atoms. Furthermore, we estimate that $n^{1 / 3}\left|a_{-}\right| \simeq 100$ in that experiment, which indicates that it is already in a regime where the nontrivial dependence on the interparticle spacing is strongly pronounced. Indeed, in a recent JILA experiment [19], the density of the Bose gas was changed by 2 orders of magnitude, and thus an experimental investigation of the density dependence of the unitary Bose polaron appears to be well within reach.

\section{ACKNOWLEDGMENTS}

We gratefully acknowledge fruitful discussions with Luis A. Peña Ardila, Jan J. Arlt, Georg M. Bruun, Rasmus S. Christensen, Victor Gurarie, Nils B. Jørgensen, Richard Schmidt, Zhe-Yu Shi, and Bing Zhu. We also thank Luis A. Peña Ardila for the QMC data of Refs. [33,36] and for helpful comments on the manuscript. S. M. Y. acknowledges support from the Japan Society for the Promotion of Science through Program for Leading Graduate Schools (ALPS) and Grant-in-Aid for JSPS Fellows (KAKENHI Grant No. JP16J06706). J. L. and M. M. P. acknowledge financial support from the Australian Research Council via Discovery Project No. DP160102739. J. L. and S. E. are supported by the Australian Research Council through Future Fellowship FT160100244 and DECRA Fellowship DE180100781, respectively. J. L. and M. M. P. acknowledge funding from the Universities Australia-Germany
Joint Research Co-operation Scheme. This work was performed in part at the Aspen Center for Physics, which is supported by the National Science Foundation Grant No. PHY-1607611.

\section{APPENDIX A: COUPLED INTEGRAL EQUATIONS}

To obtain the integral equations for the $r_{0}$ model, we first take the derivative $\langle\partial \Psi|(\hat{H}-E)| \Psi\rangle=0$ with respect to $\alpha_{0}$,

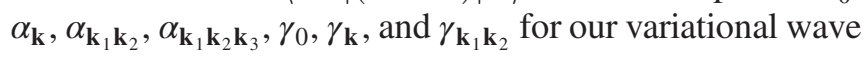
function in Eq. (5). This yields the following linear equations:

$$
\begin{gathered}
E \alpha_{0}=g \sqrt{n_{0}} \gamma_{0}-g \sum_{\mathbf{k}} v_{\mathbf{k}} \gamma_{\mathbf{k}}, \\
\left(E-\epsilon_{\mathbf{k}}-E_{\mathbf{k}}\right) \alpha_{\mathbf{k}}=g u_{\mathbf{k}} \gamma_{0}+g \sqrt{n_{0}} \gamma_{\mathbf{k}}-g \sum_{\mathbf{q}} \gamma_{\mathbf{k q}} v_{\mathbf{q}}, \quad(\mathrm{A} 2) \\
\left(E-E_{\mathbf{k}_{1} \mathbf{k}_{2}}\right) \alpha_{\mathbf{k}_{1} \mathbf{k}_{2}}=g\left(\gamma_{\mathbf{k}_{1}} u_{\mathbf{k}_{2}}+\gamma_{\mathbf{k}_{2}} u_{\mathbf{k}_{1}}\right)+g \sqrt{n_{0}} \gamma_{\mathbf{k}_{1} \mathbf{k}_{2}}, \\
\left(E-E_{\mathbf{k}_{1} \mathbf{k}_{2} \mathbf{k}_{3}}\right) \alpha_{\mathbf{k}_{1} \mathbf{k}_{2} \mathbf{k}_{3}}=g\left(\gamma_{\mathbf{k}_{1} \mathbf{k}_{2}} u_{\mathbf{k}_{3}}+\gamma_{\mathbf{k}_{2} \mathbf{k}_{3}} u_{\mathbf{k}_{1}}+\gamma_{\mathbf{k}_{1} \mathbf{k}_{3}} u_{\mathbf{k}_{2}}\right), \\
\left(E-\nu_{0}\right) \gamma_{0}=g \sqrt{n_{0}} \alpha_{0}+g \sum_{\mathbf{k}} u_{\mathbf{k}} \alpha_{\mathbf{k}}, \\
\left(E-\epsilon_{\mathbf{k}}^{d}-\nu_{0}-E_{\mathbf{k}}\right) \gamma_{\mathbf{k}}=g \sqrt{n_{0}} \alpha_{\mathbf{k}}-g v_{\mathbf{k}} \alpha_{0}+g \sum_{\mathbf{k}^{\prime}} u_{\mathbf{k}^{\prime}} \alpha_{\mathbf{k}_{\mathbf{k}^{\prime}}}, \\
\left(E-\epsilon_{\mathbf{k}_{1}+\mathbf{k}_{2}}^{d}-\nu_{0}-E_{\mathbf{k}_{1}}-E_{\mathbf{k}_{1}}\right) \gamma_{\mathbf{k}_{1} \mathbf{k}_{2}} \\
=g \sqrt{n_{0}} \alpha_{\mathbf{k}_{1} \mathbf{k}_{2}}-g\left(\alpha_{\mathbf{k}_{1}} v_{\mathbf{k}_{2}}+\alpha_{\mathbf{k}_{2}} u_{\mathbf{k}_{1}}\right)+g \sum_{\mathbf{k}_{3}} \alpha_{\mathbf{k}_{1} \mathbf{k}_{2} \mathbf{k}_{3}} u_{\mathbf{k}_{3}},
\end{gathered}
$$

where $E_{\mathbf{k}_{1} \mathbf{k}_{2}}=E_{\mathbf{k}_{1}}+E_{\mathbf{k}_{2}}+\epsilon_{\mathbf{k}_{1}+\mathbf{k}_{2}}$ and $E_{\mathbf{k}_{1} \mathbf{k}_{2} \mathbf{k}_{3}}=E_{\mathbf{k}_{1}}+$ $E_{\mathbf{k}_{2}}+E_{\mathbf{k}_{3}}+\epsilon_{\mathbf{k}_{1}+\mathbf{k}_{2}+\mathbf{k}_{3}}$. Using the first four equations to remove the $\alpha$ coefficients, we obtain

$$
\begin{aligned}
\mathcal{T}^{-1}(E, \mathbf{0}) \gamma_{0}= & \frac{n_{0}}{E} \gamma_{0}+\sqrt{n_{0}} \sum_{\mathbf{k}}\left(\frac{u_{\mathbf{k}} \gamma_{\mathbf{k}}}{E-\epsilon_{\mathbf{k}}-E_{\mathbf{k}}}-\frac{v_{\mathbf{k}} \gamma_{\mathbf{k}}}{E}\right)-\sum_{\mathbf{k q}} \frac{u_{\mathbf{k}} v_{\mathbf{q}} \gamma_{\mathbf{k q}}}{E-\epsilon_{\mathbf{k}}-E_{\mathbf{k}}}, \\
\mathcal{T}^{-1}\left(E-E_{\mathbf{k}}, \mathbf{k}\right) \gamma_{\mathbf{k}}= & \sqrt{n_{0}}\left(\frac{u_{\mathbf{k}}}{E-\epsilon_{\mathbf{k}}-E_{\mathbf{k}}}-\frac{v_{\mathbf{k}}}{E}\right) \gamma_{0}+\frac{n_{0}}{E-\epsilon_{\mathbf{k}}-E_{\mathbf{k}}} \gamma_{\mathbf{k}}+\sum_{\mathbf{q}}\left(\frac{u_{\mathbf{k}} u_{\mathbf{q}} \gamma_{\mathbf{q}}}{E-E_{\mathbf{k q}}}+\frac{v_{\mathbf{k}} v_{\mathbf{q}} \gamma_{\mathbf{q}}}{E}\right) \\
& +\sqrt{n_{0}} \sum_{\mathbf{q}}\left(\frac{u_{\mathbf{q}} \gamma_{\mathbf{k q}}}{E-E_{\mathbf{k q}}}-\frac{v_{\mathbf{q}} \gamma_{\mathbf{k q}}}{E-\epsilon_{\mathbf{k}}-E_{\mathbf{k}}}\right),
\end{aligned}
$$




$$
\begin{aligned}
\mathcal{T}^{-1}\left(E-E_{\mathbf{k}_{1}}-E_{\mathbf{k}_{2}}, \mathbf{k}_{1}+\mathbf{k}_{2}\right) \gamma_{\mathbf{k}_{1} \mathbf{k}_{2}}= & \frac{n_{0}}{E-E_{\mathbf{k}_{1} \mathbf{k}_{2}}} \gamma_{\mathbf{k}_{1} \mathbf{k}_{2}}+\left[\left\{\sqrt{n_{0}}\left(\frac{u_{\mathbf{k}_{1}} \gamma_{\mathbf{k}_{2}}}{E-E_{\mathbf{k}_{1} \mathbf{k}_{2}}}-\frac{v_{\mathbf{k}_{1}} \gamma_{\mathbf{k}_{2}}}{E-\epsilon_{\mathbf{k}_{2}}-E_{\mathbf{k}_{2}}}\right)-\frac{u_{\mathbf{k}_{1}} v_{\mathbf{k}_{2}} \gamma_{0}}{E-\epsilon_{\mathbf{k}_{1}}-E_{\mathbf{k}_{1}}}\right.\right. \\
& \left.\left.+\sum_{\mathbf{q}}\left(\frac{u_{\mathbf{k}_{1}} u_{\mathbf{q}} \gamma_{\mathbf{k}_{2} \mathbf{q}}}{E-E_{\mathbf{k}_{1} \mathbf{k}_{2} \mathbf{q}}}+\frac{v_{\mathbf{k}_{1}} v_{\mathbf{q}} \gamma_{\mathbf{k}_{2} \mathbf{q}}}{E-\epsilon_{\mathbf{k}_{2}}-E_{\mathbf{k}_{2}}}\right)\right\}+\left(\mathbf{k}_{1} \leftrightarrow \mathbf{k}_{2}\right)\right] .
\end{aligned}
$$

Here, the two-body $T$ matrix $\mathcal{T}(E, \mathbf{k})$ in the BEC medium is

$$
\begin{aligned}
\mathcal{T}^{-1}(E, \mathbf{k}) & =\frac{m}{4 \pi a}-\frac{m r_{0}}{8 \pi}\left(E-\epsilon_{\mathbf{k}}^{d}\right)-\sum_{\mathbf{q}}\left[\frac{u_{\mathbf{q}}^{2}}{E-E_{\mathbf{q}}-\epsilon_{\mathbf{k}+\mathbf{q}}}+\frac{1}{2 \epsilon_{\mathbf{q}}}\right] \\
& =\mathcal{T}_{0}^{-1}(E, \mathbf{k})+\sum_{\mathbf{q}}\left[\frac{1}{E-\epsilon_{\mathbf{q}}-\epsilon_{\mathbf{k}+\mathbf{q}}}-\frac{u_{\mathbf{q}}^{2}}{E-E_{\mathbf{q}}-\epsilon_{\mathbf{k}+\mathbf{q}}}\right],
\end{aligned}
$$

where the vacuum $T$ matrix $\mathcal{T}_{0}(E, \mathbf{k})$ was defined in Eq. (10).

The coupled integral equations for the $\Lambda$ model have the same form except that we take $r_{0} \rightarrow 0$ with $a$ fixed in the $T$ matrix and impose a UV cutoff $\Lambda$ on the integrals on the right-hand side of Eqs. (A8)-(A10). The UV cutoff is necessary because otherwise the coupled integral equations are ill posed and the three-body parameter cannot be fixed [56]. Note that the coupled integral equations with $r_{0} \rightarrow 0$ also follow from the single-channel model, which has a density-density coupling of the form $\sum_{\mathbf{k}, \mathbf{k}^{\prime}, \mathbf{q}} b_{\mathbf{k}^{\prime}}^{\dagger} c_{\mathbf{q}-\mathbf{k}^{\prime}}^{\dagger} c_{\mathbf{q}-\mathbf{k}} b_{\mathbf{k}}$ but no terms involving the closedchannel molecules. In the latter case, we again need to introduce a finite cutoff $\Lambda$ by hand to have a fixed three-body parameter.

In this paper, we show results obtained from variational wave functions, which include 1,2, or 3 excitations of the condensate; see Eq. (6). These approximations correspond to solving Eq. (A8) with $\gamma_{\mathbf{k}}=\gamma_{\mathbf{k q}}=0$, Eqs. (A8) and (A9) with $\gamma_{\mathbf{k q}}=0$, and all of Eqs. (A8)-(A10), respectively.

\section{APPENDIX B: EFFECT OF FINITE COHERENCE LENGTH AND NUMBER OF BOGOLIUBOV EXCITATIONS}

In Sec. IV C, we compared the results of our variational approach with those obtained in quantum Monte Carlo calculations by Peña Ardila and Giorgini [36], and we found very good agreement across more than an order of magnitude in interparticle spacing (see Fig. 2). In this comparison, we highlighted the fact that the dependence of the polaron energy on the three-body parameter $a_{-}$is much stronger than the dependence on $a_{B}$, and to emphasize this, we took $a_{B}=0$. However, as we have argued in Sec. III, in the QMC calculation the ratio between $a_{-}$and $a_{B}$ was kept fixed at a value of $a_{-}^{(\mathrm{QMC})} \simeq-2.09(36) \times 10^{4} a_{B}^{(\mathrm{QMC})}$ as identified in Eq. (12). Therefore, we now consider the residual effect of keeping $a_{-} / a_{B}$ fixed in the same manner as in the QMC. Our results for the polaron energy within the $\Lambda$ model are shown in Fig. 8. We see that the polaron energy is almost unaffected in the low-density, few-body dominated regime, while $a_{B}$ becomes more important with increasing density. However, throughout the range plotted, i.e., the universal many-body regime, the relative change in energy arising from the change in $n^{1 / 3}\left|a_{-}\right|$clearly greatly exceeds that from the increase in $n^{1 / 3} a_{B}$. We furthermore see that both sets of results are consistent with our reinterpretation of the QMC results in terms of a threebody parameter.

In the inset of Fig. 8, we furthermore illustrate the convergence of the polaron energy calculated within both interaction models as a function of (inverse) number of

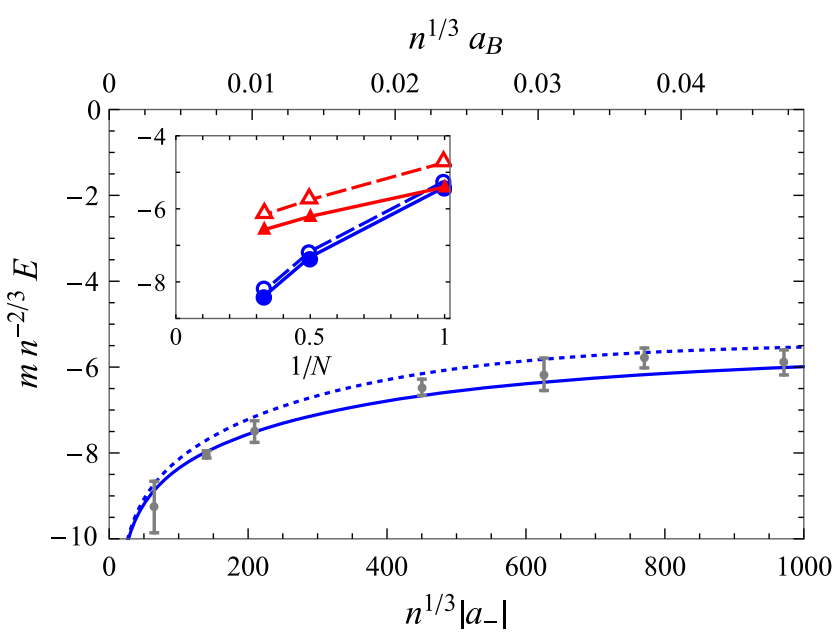

FIG. 8. Polaron energy calculated within the $\Lambda$ model as a function of $n^{1 / 3}\left|a_{-}\right|$for $a_{B}=0$ (solid line) and for $a_{B}=$ $2.1 \times 10^{4}\left|a_{-}\right|$(dashed line). The latter choice of $a_{B}$ ensures that we take the same ratio of $a_{-}$and $a_{B}$ as in the QMC calculation (gray dots) [see Eq. (12)], and the corresponding scale for $a_{B}$ is shown on the top $x$ axis. Inset: Polaron energy at unitarity as a function of inverse number $N$ of Bogoliubov excitations for $a_{B}=0$. We present the results of the $\Lambda$ model (solid) and $r_{0}$ model (open) for $n^{1 / 3}\left|a_{-}\right|=100$ (blue circles) and $n^{1 / 3}\left|a_{-}\right|=$ 500 (red triangles). 
Bogoliubov excitations included in our variational approach. In the many-body dominated regime, at $n^{1 / 3}\left|a_{-}\right|=500$, we see that the convergence appears to be nearly linear, with a slope (and extrapolation to an infinite number of excitations) that is consistent with the difference between the finite $a_{B}$ dotted line and the QMC results in the main figure. Closer to the few-body dominated regime at $n^{1 / 3}\left|a_{-}\right|=100$, our approach converges more slowly because of the strong few-body correlations induced by the trimer and tetramer bound states. However, it is likely that including four Bogoliubov excitations in our approach to account for the hypothesized pentamer (see Sec. IVA) would yield a nearly converged result. We expect a small residual difference between the results of the two interaction models, even in the $N \rightarrow \infty$ limit, because of the different manners in which the two-body physics is implemented. A similar effect would be present for an impurity in a Fermi gas.

\section{APPENDIX C: CALCULATION OF OBSERVABLES}

Once the wave function $|\Psi\rangle$ is obtained, we can calculate physical quantities of interest. In the following, we assume that the variational wave function is normalized, $\langle\Psi \mid \Psi\rangle=1$. When we solve the renormalized equations (A8)-(A10), this implies that

$$
\begin{aligned}
1= & \left|\alpha_{0}\right|^{2}+\sum_{\mathbf{k}}\left|\alpha_{\mathbf{k}}\right|^{2}+\frac{1}{2} \sum_{\mathbf{k}_{1} \mathbf{k}_{2}}\left|\alpha_{\mathbf{k}_{1} \mathbf{k}_{2}}\right|^{2} \\
& +\frac{1}{6} \sum_{\mathbf{k}_{1} \mathbf{k}_{2} \mathbf{k}_{3}}\left|\alpha_{\mathbf{k}_{1} \mathbf{k}_{2} \mathbf{k}_{3}}\right|^{2}+\left|\gamma_{0}\right|^{2}+\sum_{\mathbf{k}}\left|\gamma_{\mathbf{k}}\right|^{2}+\frac{1}{2} \sum_{\mathbf{k}_{1} \mathbf{k}_{2}}\left|\gamma_{\mathbf{k}_{1} \mathbf{k}_{2}}\right|^{2} .
\end{aligned}
$$

Since we solve for $\gamma_{0}, \gamma_{\mathbf{k}}$, and $\gamma_{\mathbf{k}_{1} \mathbf{k}_{2}}$, we relate the $\alpha$ coefficients to these through Eqs. (A1)-(A4).

\section{Effective mass}

For a system with rotational symmetry, the inverse effective mass is defined as

$$
\frac{1}{m^{*}}=\frac{\partial^{2} E(P)}{\partial P^{2}},
$$

where $E(P)$ is the energy dispersion of the Bose polaron. The variational ansatz can be easily extended to a Bose polaron with a finite momentum, but solving the resulting integral equations becomes numerically expensive because the equations do not have rotational symmetry. Here, following a line of argument similar to Ref. [90], we present a method to calculate the effective mass without solving the integral equations for a finite-momentum Bose polaron.

In general, the coupled integral equations for $\gamma$ 's for a finite-momentum polaron have the following structure [similar to Eqs. (A1)-(A4)]:

$$
\hat{X}[E(P), \mathbf{P}]|\gamma\rangle=0 .
$$

Here, $|\gamma\rangle$ is an ordered set of $\gamma_{0}, \gamma_{\mathbf{k}}$, and $\gamma_{\mathbf{k}_{1} \mathbf{k}_{2}}$, and the center-of-mass momentum can be taken to be $P \mathbf{e}_{z}$ without loss of generality. While the matrix $\hat{X}$ is not the Hamiltonian, it is still Hermitian. The energy dispersion $E(P)$ is determined by the zero crossing of the lowest (or highest, depending on the sign convention) eigenvalue of $\hat{X}$. Therefore, for a small $P$, we can expand $\hat{X}$ up to $\mathcal{O}\left(P^{2}\right)$ and evaluate the lowest eigenvalue in the same way as the Rayleigh-Schrödinger perturbation theory. It is straightforward to show that

$$
\begin{aligned}
\hat{X}[E(P), P]= & \hat{X}_{0}+P \hat{X}_{P}+\frac{P^{2}}{2}\left(\hat{X}_{P P}+\left(1 / m^{*}\right) \hat{X}_{E}\right) \\
& +\mathcal{O}\left(P^{3}\right),
\end{aligned}
$$

where

$$
\begin{gathered}
\hat{X}_{0}=\hat{X}[E(0), 0], \quad \hat{X}_{P}=\left.\frac{\partial \hat{X}}{\partial P}[E, P]\right|_{\substack{P=0 \\
E=E(0)}}, \\
\hat{X}_{P P}=\left.\frac{\partial^{2} \hat{X}}{\partial P^{2}}[E, P]\right|_{\substack{P=0 \\
E=E(0)}}, \quad \hat{X}_{E}=\left.\frac{\partial \hat{X}}{\partial E}[E, P]\right|_{\substack{P=0 \\
E=E(0)}} .
\end{gathered}
$$

Then, the lowest eigenvalue $\lambda(P)$ of $\hat{X}[E(P), P]$ can be expressed as a series expansion in terms of $P$,

$$
\lambda(P)=\lambda_{0}+\lambda_{1} P+\lambda_{2} P^{2}+\mathcal{O}\left(P^{3}\right),
$$

and its coefficients $\lambda_{i}$ are determined perturbatively:

$$
\lambda_{1}=\left\langle\gamma^{(0)}\left|\hat{X}_{P}\right| \gamma^{(0)}\right\rangle=0,
$$

$\lambda_{2}=\frac{1}{2}\left\langle\gamma^{(0)}\left|\left(\hat{X}_{P P}+\left(1 / m^{*}\right) \hat{X}_{E}\right)\right| \gamma^{(0)}\right\rangle+\sum_{i>0} \frac{\left|\left\langle\gamma^{(i)}\left|\hat{X}_{P}\right| \gamma^{(0)}\right\rangle\right|^{2}}{\lambda_{0}^{(0)}-\lambda_{0}^{(i)}}$.

Here, $\lambda_{0}^{(i)}$ and $\left|\gamma^{(i)}\right\rangle$ are the $i$ th eigenvalue and its corresponding eigenvector of $\hat{X}_{0}$, and $i=0$ corresponds to the state that satisfies $\hat{X}_{0}[E(0), \mathbf{0}]\left|\gamma^{(0)}\right\rangle=\lambda_{0}^{(0)}\left|\gamma^{(0)}\right\rangle=0$. Note that $\lambda_{1}=0$ follows from the rotational invariance of $\left|\gamma^{(0)}\right\rangle$. Now, recalling that the energy dispersion is found by zero crossing of the lowest eigenvalue of $\hat{X}[E(P), P]$, we can set

$$
\lambda(P)=\lambda_{0}^{(0)}=0,
$$

which, in turn, implies that $\lambda_{2}=0$. From this, the inverse effective mass is obtained as follows: 


$$
\begin{aligned}
1 / m^{*}= & \left\langle\gamma^{(0)}\left|\hat{X}_{E}\right| \gamma^{(0)}\right\rangle^{-1} \\
& \times\left[\sum_{i>0} \frac{2}{\lambda_{0}^{(i)}}\left|\left\langle\gamma^{(i)}\left|\hat{X}_{P}\right| \gamma^{(0)}\right\rangle\right|^{2}-\left\langle\gamma^{(0)}\left|\hat{X}_{P P}\right| \gamma^{(0)}\right\rangle\right] \\
= & \left\langle\gamma^{(0)}\left|\hat{X}_{E}\right| \gamma^{(0)}\right\rangle^{-1}\left[2\left\langle\gamma^{(0)}\left|\hat{X}_{P} \hat{Q} \hat{X}_{0}^{-1} \hat{Q} \hat{X}_{P}\right| \gamma^{(0)}\right\rangle\right. \\
& \left.-\left\langle\gamma^{(0)}\left|\hat{X}_{P P}\right| \gamma^{(0)}\right\rangle\right],
\end{aligned}
$$

where $\hat{Q}=1-\sum_{i>0}\left|\gamma^{(i)}\right\rangle\left\langle\gamma^{(i)}\right|$.

[1] M. Randeria, W. Zwerger, and M. Zwierlein, in The BCSBEC Crossover and the Unitary Fermi Gas, edited by W. Zwerger (Springer, Berlin, Heidelberg, 2012), pp. 1-32.

[2] M. J. Ku, A. T. Sommer, L. W. Cheuk, and M. W. Zwierlein, Revealing the Superfluid Lambda Transition in the Universal Thermodynamics of a Unitary Fermi Gas, Science 335, 563 (2012).

[3] S. Nascimbène, N. Navon, K. Jiang, F. Chevy, and C. Salomon, Exploring the Thermodynamics of a Universal Fermi Gas, Nature (London) 463, 1057 (2010).

[4] M. Horikoshi, S. Nakajima, M. Ueda, and T. Mukaiyama, Measurement of Universal Thermodynamic Functions for a Unitary Fermi Gas, Science 327, 442 (2010).

[5] G. B. Partridge, K. E. Strecker, R. I. Kamar, M. W. Jack, and R. G. Hulet, Molecular Probe of Pairing in the BEC-BCS Crossover, Phys. Rev. Lett. 95, 020404 (2005).

[6] J. T. Stewart, J. P. Gaebler, and D. S. Jin, Using Photoemission Spectroscopy to Probe a Strongly Interacting Fermi Gas, Nature (London) 454, 744 (2008).

[7] A. Schirotzek, Y.-i. Shin, C. H. Schunck, and W. Ketterle, Determination of the Superfluid Gap in Atomic Fermi Gases by Quasiparticle Spectroscopy, Phys. Rev. Lett. 101, 140403 (2008).

[8] Y. Inada, M. Horikoshi, S. Nakajima, M. KuwataGonokami, M. Ueda, and T. Mukaiyama, Critical Temperature and Condensate Fraction of a Fermion Pair Condensate, Phys. Rev. Lett. 101, 180406 (2008).

[9] G. Veeravalli, E. Kuhnle, P. Dyke, and C. J. Vale, Bragg Spectroscopy of a Strongly Interacting Fermi Gas, Phys. Rev. Lett. 101, 250403 (2008).

[10] N. Navon, S. Nascimbène, F. Chevy, and C. Salomon, The Equation of State of a Low-Temperature Fermi Gas with Tunable Interactions, Science 328, 729 (2010).

[11] J. T. Stewart, J.P. Gaebler, T. E. Drake, and D. S. Jin, Verification of Universal Relations in a Strongly Interacting Fermi Gas, Phys. Rev. Lett. 104, 235301 (2010).

[12] E. D. Kuhnle, H. Hu, X.-J. Liu, P. Dyke, M. Mark, P. D. Drummond, P. Hannaford, and C. J. Vale, Universal Behavior of Pair Correlations in a Strongly Interacting Fermi Gas, Phys. Rev. Lett. 105, 070402 (2010).

[13] C. Cao, E. Elliott, J. Joseph, H. Wu, J. Petricka, T. Schäfer, and J.E. Thomas, Universal Quantum Viscosity in a Unitary Fermi Gas, Science 331, 58 (2011).
[14] P. Makotyn, C. E. Klauss, D. L. Goldberger, E. A. Cornell, and D. S. Jin, Universal Dynamics of a Degenerate Unitary Bose Gas, Nat. Phys. 10, 116 (2014).

[15] M.-G. Hu, M. J. Van de Graaff, D. Kedar, J. P. Corson, E. A. Cornell, and D.S. Jin, Bose Polarons in the Strongly Interacting Regime, Phys. Rev. Lett. 117, 055301 (2016).

[16] N. B. Jørgensen, L. Wacker, K. T. Skalmstang, M. M. Parish, J. Levinsen, R. S. Christensen, G. M. Bruun, and J. J. Arlt, Observation of Attractive and Repulsive Polarons in a Bose-Einstein Condensate, Phys. Rev. Lett. 117, 055302 (2016).

[17] R. J. Fletcher, R. Lopes, J. Man, N. Navon, R. P. Smith, M. W. Zwierlein, and Z. Hadzibabic, Two- and Three-Body Contacts in the Unitary Bose Gas, Science 355, 377 (2017).

[18] R. Lopes, C. Eigen, A. Barker, K. G. H. Viebahn, M. Robert-de Saint-Vincent, N. Navon, Z. Hadzibabic, and R. P. Smith, Quasiparticle Energy in a Strongly Interacting Homogeneous Bose-Einstein Condensate, Phys. Rev. Lett. 118, 210401 (2017).

[19] C. E. Klauss, X. Xie, C. Lopez-Abadia, J. P. D’Incao, Z. Hadzibabic, D. S. Jin, and E. A. Cornell, Observation of Efimov Molecules Created from a Resonantly Interacting Bose Gas, Phys. Rev. Lett. 119, 143401 (2017).

[20] C. Eigen, J. A. P. Glidden, R. Lopes, N. Navon, Z. Hadzibabic, and R.P. Smith, Universal Scaling Laws in the Dynamics of a Homogeneous Unitary Bose Gas, Phys. Rev. Lett. 119, 250404 (2017).

[21] V. Efimov, Energy Levels Arising from Resonant Two-Body Forces in a Three-Body System, Phys. Lett. B 33, 563 (1970).

[22] E. Braaten and H.-W. Hammer, Universality in Few-Body Systems with Large Scattering Length, Phys. Rep. 428, 259 (2006).

[23] T. Kraemer, M. Mark, P. Waldburger, J. G. Danzl, C. Chin, B. Engeser, A. D. Lange, K. Pilch, A. Jaakkola, H.-C. Nägerl, and R. Grimm, Evidence for Efimov Quantum States in an Ultracold Gas of Caesium Atoms, Nature (London) 440, 315 (2006).

[24] P. Naidon and S. Endo, Efimov Physics: A Review, Rep. Prog. Phys. 80, 056001 (2017).

[25] C. H. Greene, P. Giannakeas, and J. Pérez-Ríos, Universal Few-Body Physics and Cluster Formation, Rev. Mod. Phys. 89, 035006 (2017).

[26] J. P. D'Incao, Few-Body Physics in Resonantly Interacting Ultracold Quantum Gases, arXiv:1705.10860.

[27] J. Tempere, W. Casteels, M. K. Oberthaler, S. Knoop, E. Timmermans, and J. T. Devreese, Feynman Path-Integral Treatment of the BEC-Impurity Polaron, Phys. Rev. B 80, 184504 (2009).

[28] S. P. Rath and R. Schmidt, Field-Theoretical Study of the Bose Polaron, Phys. Rev. A 88, 053632 (2013).

[29] W. Li and S. Das Sarma, Variational Study of Polarons in Bose-Einstein Condensates, Phys. Rev. A 90, 013618 (2014).

[30] A. Shashi, F. Grusdt, D. A. Abanin, and E. Demler, Radio-Frequency Spectroscopy of Polarons in Ultracold Bose Gases, Phys. Rev. A 89, 053617 (2014).

[31] R.S. Christensen, J. Levinsen, and G. M. Bruun, Quasiparticle Properties of a Mobile Impurity in a BoseEinstein Condensate, Phys. Rev. Lett. 115, 160401 (2015). 
[32] J. Levinsen, M. M. Parish, and G. M. Bruun, Impurity in a Bose-Einstein Condensate and the Efimov Effect, Phys. Rev. Lett. 115, 125302 (2015).

[33] L. A. Peña Ardila and S. Giorgini, Impurity in a BoseEinstein Condensate: Study of the Attractive and Repulsive Branch Using Quantum Monte Carlo Methods, Phys. Rev. A 92, 033612 (2015).

[34] F. Grusdt, Y. E. Shchadilova, A. N. Rubtsov, and E. Demler, Renormalization Group Approach to the Fröhlich Polaron Model: Application to Impurity-BEC Problem, Sci. Rep. 5, 12124 (2015).

[35] F. Grusdt and E. A. Demler, New Theoretical Approaches to Bose Polarons, arXiv:1510.04934.

[36] L. A. Peña Ardila and S. Giorgini, Bose Polaron Problem: Effect of Mass Imbalance on Binding Energy, Phys. Rev. A 94, 063640 (2016).

[37] Y. E. Shchadilova, R. Schmidt, F. Grusdt, and E. Demler, Quantum Dynamics of Ultracold Bose Polarons, Phys. Rev. Lett. 117, 113002 (2016).

[38] N. J. S. Loft, Z. Wu, and G. M. Bruun, Mixed-Dimensional Bose Polaron, Phys. Rev. A 96, 033625 (2017).

[39] F. Grusdt, R. Schmidt, Y. E. Shchadilova, and E. Demler, Strong-Coupling Bose Polarons in a Bose-Einstein Condensate, Phys. Rev. A 96, 013607 (2017).

[40] M. Sun, H. Zhai, and X. Cui, Visualizing the Efimov Correlation in Bose Polarons, Phys. Rev. Lett. 119, 013401 (2017).

[41] A. Lampo, S. H. Lim, M. Á. García-March, and M. Lewenstein, Bose Polaron as an Instance of Quantum Brownian Motion, Quantum 1, 30 (2017).

[42] R. Schmidt and M. Lemeshko, Rotation of Quantum Impurities in the Presence of a Many-Body Environment, Phys. Rev. Lett. 114, 203001 (2015).

[43] R. Schmidt, H. R. Sadeghpour, and E. Demler, Mesoscopic Rydberg Impurity in an Atomic Quantum Gas, Phys. Rev. Lett. 116, 105302 (2016).

[44] F. Camargo, R. Schmidt, J. D. Whalen, R. Ding, G. Woehl, Jr., S. Yoshida, J. Burgdörfer, F. B. Dunning, H. R. Sadeghpour, E. Demler, and T. C. Killian, Creation of Rydberg Polarons in a Bose Gas, arXiv:1706.03717.

[45] T. Rentrop, A. Trautmann, F. A. Olivares, F. Jendrzejewski, A. Komnik, and M. K. Oberthaler, Observation of the Phononic Lamb Shift with a Synthetic Vacuum, Phys. Rev. X 6, 041041 (2016).

[46] Y. Wang, J. Wang, J. P. D'Incao, and C. H. Greene, Universal Three-Body Parameter in Heteronuclear Atomic Systems, Phys. Rev. Lett. 109, 243201 (2012).

[47] D. Blume and Y. Yan, Generalized Efimov Scenario for Heavy-Light Mixtures, Phys. Rev. Lett. 113, 213201 (2014).

[48] F. Chevy, Universal Phase Diagram of a Strongly Interacting Fermi Gas with Unbalanced Spin Populations, Phys. Rev. A 74, 063628 (2006).

[49] R. Combescot and S. Giraud, Normal State of Highly Polarized Fermi Gases: Full Many-Body Treatment, Phys. Rev. Lett. 101, 050404 (2008).

[50] N. Prokof'ev and B. Svistunov, Fermi-Polaron Problem: Diagrammatic Monte Carlo Method for Divergent SignAlternating Series, Phys. Rev. B 77, 020408 (2008).
[51] J. Vlietinck, J. Ryckebusch, and K. Van Houcke, Quasiparticle Properties of an Impurity in a Fermi Gas, Phys. Rev. B 87, 115133 (2013).

[52] P. Massignan, M. Zaccanti, and G. M. Bruun, Polarons, Dressed Molecules and Itinerant Ferromagnetism in Ultracold Fermi Gases, Rep. Prog. Phys. 77, 034401 (2014).

[53] P. W. Anderson, Infrared Catastrophe in Fermi Gases with Local Scattering Potentials, Phys. Rev. Lett. 18, 1049 (1967).

[54] A. L. Fetter and J. D. Walecka, Quantum Theory of ManyParticle Systems (McGraw-Hill, New York, 1971).

[55] D. S. Petrov, Three-Boson Problem Near a Narrow Feshbach Resonance, Phys. Rev. Lett. 93, 143201 (2004).

[56] P. Bedaque, H.-W. Hammer, and U. van Kolck, The ThreeBoson System with Short-Range Interactions, Nucl. Phys. A646, 444 (1999).

[57] E. Timmermans, P. Tommasini, M. Hussein, and A. Kerman, Feshbach Resonances in Atomic Bose-Einstein Condensates, Phys. Rep. 315, 199 (1999).

[58] G. M. Bruun and C. J. Pethick, Effective Theory of Feshbach Resonances and Many-Body Properties of Fermi Gases, Phys. Rev. Lett. 92, 140404 (2004).

[59] J. Levinsen and D. Petrov, Atom-Dimer and Dimer-Dimer Scattering in Fermionic Mixtures Near a Narrow Feshbach Resonance, Eur. Phys. J. D 65, 67 (2011).

[60] M. M. Parish and J. Levinsen, Quantum Dynamics of Impurities Coupled to a Fermi Sea, Phys. Rev. B 94, 184303 (2016).

[61] L. Pricoupenko, Isotropic Contact Forces in Arbitrary Representation: Heterogeneous Few-Body Problems and Low Dimensions, Phys. Rev. A 83, 062711 (2011).

[62] V. Efimov, Energy Levels of Three Resonantly Interacting Particles, Nucl. Phys. A210, 157 (1973).

[63] H.-W. Hammer and L. Platter, Universal Properties of the Four-Body System with Large Scattering Length, Eur. Phys. J. A 32, 113 (2007).

[64] J. von Stecher, J. P. D'Incao, and C. H. Greene, Signatures of Universal Four-Body Phenomena and Their Relation to the Efimov Effect, Nat. Phys. 5, 417 (2009).

[65] M. R. Hadizadeh, M. T. Yamashita, L. Tomio, A. Delfino, and T. Frederico, Scaling Properties of Universal Tetramers, Phys. Rev. Lett. 107, 135304 (2011).

[66] A. Deltuva, Shallow Efimov Tetramer as Inelastic Virtual State and Resonant Enhancement of the Atom-Trimer Relaxation, Europhys. Lett. 95, 43002 (2011).

[67] F. Ferlaino, S. Knoop, M. Berninger, W. Harm, J. P. D'Incao, H.-C. Nägerl, and R. Grimm, Evidence for Universal Four-Body States Tied to an Efimov Trimer, Phys. Rev. Lett. 102, 140401 (2009).

[68] Y. Wang, W. B. Laing, J. von Stecher, and B. D. Esry, Efimov Physics in Heteronuclear Four-Body Systems, Phys. Rev. Lett. 108, 073201 (2012).

[69] C. H. Schmickler, H.-W. Hammer, and E. Hiyama, Tetramer Bound States in Heteronuclear Systems, Phys. Rev. A 95, 052710 (2017).

[70] We have not investigated tetramer states tied to the excited Efimov trimer, but we expect that they will exist as resonant states embedded in the atom-trimer continuum, as is the case for identical bosons [66,71]. 
[71] J. von Stecher, Five- and Six-Body Resonances Tied to an Efimov Trimer, Phys. Rev. Lett. 107, 200402 (2011).

[72] This number had a typo in Ref. [32], which did not affect any of the other results of that paper.

[73] B. Huang, L. A. Sidorenkov, R. Grimm, and J. M. Hutson, Observation of the Second Triatomic Resonance in Efimov's Scenario, Phys. Rev. Lett. 112, 190401 (2014).

[74] The exception to this expected universality is the crossing of the ground-state trimer with the atom-dimer continuum (not shown in Fig. 1). This crossing is, in general, known to be the least universal aspect of Efimov trimers, as it corresponds to the largest energy scale. We indeed find that the ground-state trimer crossing is at $a_{*} /\left|a_{-}\right| \simeq 4.86 \times 10^{-4}$ in the $r_{0}$ model and at $a_{*} /\left|a_{-}\right| \simeq 5.58 \times 10^{-4}$ in the $\Lambda$ model, neither of which quite fits the universal prediction: $a_{*} /\left|a_{-}\right| \simeq 0.72418 e^{-\pi / s_{0}}=3.6 \times 10^{-4}$ [75]. On the other hand, we find that the first excited trimer, with $a_{*}^{(1)} /\left|a_{-}\right| \simeq$ 0.71 , matches reasonably well.

[75] K. Helfrich, H.-W. Hammer, and D. S. Petrov, Three-Body Problem in Heteronuclear Mixtures with Resonant Interspecies Interaction, Phys. Rev. A 81, 042715 (2010).

[76] Under the assumption of universality where the quantity $m a_{-}^{2} E_{4 B,-}^{(1)}$ is the same in all models, the ratio is given by $a_{-}^{(\mathrm{QMC})} / a_{B}^{(\mathrm{QMC})} \simeq \sqrt{r_{0}^{2} E_{4 B,-}^{(1,2 \mathrm{ch})} / a_{B}^{2} E_{4 B,-}^{(1, \mathrm{QMC})}} a_{-}^{(2 \mathrm{ch})} / r_{0}^{(2 \mathrm{ch})}$. All parameters on the right-hand side are known from the results of the QMC and the $r_{0}$ model.

[77] M. Berninger, A. Zenesini, B. Huang, W. Harm, H.-C. Nägerl, F. Ferlaino, R. Grimm, P. S. Julienne, and J. M. Hutson, Universality of the Three-Body Parameter for Efimov States in Ultracold Cesium, Phys. Rev. Lett. 107, 120401 (2011).

[78] J. Wang, J. P. D’Incao, B. D. Esry, and C. H. Greene, Origin of the Three-Body Parameter Universality in Efimov Physics, Phys. Rev. Lett. 108, 263001 (2012).

[79] P. Naidon, S. Endo, and M. Ueda, Physical Origin of the Universal Three-Body Parameter in Atomic Efimov Physics, Phys. Rev. A 90, 022106 (2014).

[80] P. Naidon, S. Endo, and M. Ueda, Microscopic Origin and Universality Classes of the Efimov Three-Body Parameter, Phys. Rev. Lett. 112, 105301 (2014).

[81] Y. Wang and P.S. Julienne, Universal van der Waals Physics for Three Cold Atoms Near Feshbach Resonances, Nat. Phys. 10, 768 (2014).

[82] J. Von Stecher, Weakly Bound Cluster States of Efimov Character, J. Phys. B 43, 101002 (2010).

[83] M. Gattobigio and A. Kievsky, Universality and Scaling in the N-Body Sector of Efimov Physics, Phys. Rev. A 90, 012502 (2014).

[84] Y. Yan and D. Blume, Energy and Structural Properties of N-Boson Clusters Attached to Three-Body Efimov States: Two-Body Zero-Range Interactions and the Role of the Three-Body Regulator, Phys. Rev. A 92, 033626 (2015).

[85] A. Zenesini, B. Huang, M. Berninger, S. Besler, H.-C. Nägerl, F. Ferlaino, R. Grimm, C. H. Greene, and
J. von Stecher, Resonant Five-Body Recombination in an Ultracold Gas of Bosonic Atoms, New J. Phys. 15, 043040 (2013).

[86] B. Bazak and D. S. Petrov, Five-Body Efimov Effect and Universal Pentamer in Fermionic Mixtures, Phys. Rev. Lett. 118, 083002 (2017).

[87] B. Bazak, Mass-Imbalanced Fermionic Mixture in a Harmonic Trap, Phys. Rev. A 96, 022708 (2017).

[88] V. Gurarie and L. Radzihovsky, Resonantly Paired Fermionic Superfluids, Ann. Phys. (Amsterdam) 322, 2 (2007).

[89] P. Massignan, Polarons and Dressed Molecules Near Narrow Feshbach Resonances, Europhys. Lett. 98, 10012 (2012).

[90] C. Trefzger and Y. Castin, Impurity in a Fermi Sea on a Narrow Feshbach Resonance: A Variational Study of the Polaronic and Dimeronic Branches, Phys. Rev. A 85, 053612 (2012).

[91] R. Qi and H. Zhai, Highly Polarized Fermi Gases across a Narrow Feshbach Resonance, Phys. Rev. A 85, 041603 (2012).

[92] C. Kohstall, M. Zaccanti, M. Jag, A. Trenkwalder, P. Massignan, G. M. Bruun, F. Schreck, and R. Grimm, Metastability and Coherence of Repulsive Polarons in a Strongly Interacting Fermi Mixture, Nature (London) $\mathbf{4 8 5}$, 615 (2012).

[93] M. Cetina, M. Jag, R. S. Lous, I. Fritsche, J. T. M. Walraven, R. Grimm, J. Levinsen, M. M. Parish, R. Schmidt, M. Knap, and E. Demler, Ultrafast Many-Body Interferometry of Impurities Coupled to a Fermi Sea, Science 354, 96 (2016).

[94] A. Schirotzek, C.-H. Wu, A. Sommer, and M. W. Zwierlein, Observation of Fermi Polarons in a Tunable Fermi Liquid of Ultracold Atoms, Phys. Rev. Lett. 102, 230402 (2009).

[95] The high-density expansion for $Z$ at finite $a_{B}$ can, in principle, be calculated using an approach similar to that in Ref. [31]. However, such a lengthy perturbative analysis goes beyond the scope of this work.

[96] P. W. Anderson, Infrared Catastrophe: When Does It Trash Fermi Liquid Theory?, in The Hubbard Model. NATO ASI Series (Series B: Physics), Vol. 343, edited by D. Baeriswyl, D. K. Campbell, J. M. P. Carmelo, F. Guinea, and E. Louis (Springer, Boston, MA, 1995), p. 217.

[97] S. Tan, Energetics of a Strongly Correlated Fermi Gas, Ann. Phys. (Amsterdam) 323, 2952 (2008); Large Momentum Part of Fermions with Large Scattering Length, Ann. Phys. (Amsterdam) 323, 2971 (2008); Generalized Virial Theorem and Pressure Relation for a Strongly Correlated Fermi Gas, Ann. Phys. (Amsterdam) 323, 2987 (2008).

[98] E. Braaten, D. Kang, and L. Platter, Universal Relations for a Strongly Interacting Fermi Gas Near a Feshbach Resonance, Phys. Rev. A 78, 053606 (2008).

[99] Y. E. Shchadilova, F. Grusdt, A. N. Rubtsov, and E. Demler, Polaronic Mass Renormalization of Impurities in Bose-Einstein Condensates: Correlated Gaussian-WaveFunction Approach, Phys. Rev. A 93, 043606 (2016). 